\title{
Simulation of turbulent lifted methane jet flames: effects of air-dilution and transient flame propagation
}

\author{
Z. Chen, S. Ruan, N. Swaminathan* \\ Department of Engineering, Cambridge University, Cambridge CB2 1PZ, UK \\ (Accepted for publication in Combust. Flame, Sep. 2014)
}

\begin{abstract}
Turbulent lifted methane jet flames with various air-dilution levels and a range of inlet velocities are simulated. A partially premixed combustion model based on premixed flamelets with presumed joint Probability Density Function (PDF) is used. The joint PDF is obtained using a copula to include the statistical correlation between mixture fraction, $Z$, and progress variable, $c$. The non-premixed combustion effect is included using a simple algebraic model. Both steady and unsteady RANS simulations are performed. The steady simulations show that the computed lift-off heights agree well with measured values for a wide range of jet velocities and air-dilution level. Both of the Z-c correlation and non-premixed combustion effects are found to be important to get the correct lift-off height. Their individual and combined effects are analysed systematically. The unsteady RANS results indicate that multi-stage flame development, namely the initial expansion, flame brush development, its propagation and final stabilisation, is captured reasonably well in simulations. The various stages of temporal evolution of the flame brush
\end{abstract}

\footnotetext{
*Corresponding author.

Email address: ns341@cam. ac.uk (N. Swaminathan)
} 
edge is captured well and the agreement with experimental measurements is good. Keywords: Partially premixed combustion; Correlated joint PDF; Air dilution; Flame lift-off height; Flame stabilisation.

\section{Introduction}

Turbulent lifted flames are quite common in many practical devices such as aero engines, gas flares, etc. These flames have been investigated in the past using analytical methods [1, 2], experiments [3-11] and numerical simulations [12-19]. These studies are reviewed critically in [20-24] highlighting the importance of this topic and challenges involved in computing these flames.

The flame stabilisation at the base of a turbulent lifted flames involves a fine balance among many complex physical processes such as partial premixing between fuel jet and entrained ambient air, flame propagation [4], interaction between flame leading edge and large-scale flow structure [2, 25, 26], edge-flame propagation [14], triple-flames [11, 15, 27, 28] and possibly extinction of nonpremixed flamelets due to high scalar dissipation rate near the leading edge [1]. Autoignition [26, 29-31] plays an important role when there is a heated co-flow with sufficiently large temperature surrounding the fuel jet. These complexities offer considerable challenge in modelling turbulent lifted flames. These flames without hot co-flow, which is of interest for this study, have been modelled in the past using various methodologies, such as the $G$-equation or level-set approach [13, 21, 32], flamelet models involving premixed and non-premixed flamelets [12, 17, 18, 33-37] and Conditional Moment Closure (CMC) [16, 38, 39]. These flames have also been computed using large eddy simulation methodology [17, 18, 40]. 
For this study, the flamelet based approaches for Reynolds averaged NavierStokes (RANS) simulations of turbulent lifted jet flames are of specific interest. The use of premixed flamelets to model these flames was suggested by Bradley and his co-workers [12, 33]. In this mixedness-reactedness flamelets approach, a range of premixed flamelets covering the entire range of flammable mixture fraction were combined to get the mean reaction rate using

$$
\overline{\dot{\omega}}=\int_{0}^{1} \int_{0}^{1} \dot{\omega}(\zeta, \xi) P(\zeta, \xi) d \zeta d \xi,
$$

where $\zeta$ is the sample space variable for a reaction progress variable, $c$, and $\xi$ is the sample space variable for the mixture fraction, $Z$. The reactedness is denoted by $c$ and mixedness is denoted by $Z$. The joint probability density function (JPDF), $P(\zeta, \xi)$, was generally taken to be a product of two marginal PDFs in past studies. Equation (1) can also be employed to get filtered reaction rate for LES, but the JPDF must be seen as the sub-grid PDF. The lift-off heights computed using this modelling approach in RANS calculations [12, 33] compared well with measured values [8, 41] for jet velocities ranging from 40 to $100 \mathrm{~m} / \mathrm{s}$ whereas the agreement was not as good for velocities ranging from 18 to $38 \mathrm{~m} / \mathrm{s}$. Since the rate of entraining surrounding air depends strongly on the jet velocity, the premixing level is expected to be low for low jet velocities and thus it is not unexpected that the comparison of lift-off heights computed using only premixed flamelets for lower jet velocities were not as good as for higher velocities. The role of radiative heat loss on the lift-off height was also assessed to be negligible [34] using the above mixedness-reactedness flamelets. Using an alternative approach involving diffusion flamelets, proposed originally in [1], Müller et al. [13] used $G$-equation involving the concept of turbulent burning velocity, $S_{\mathrm{T}}$. This allowed 
Müller et al. to include contributions of premixed flame propagation, effects of partial premixing and flamelets quenching on $S_{\mathrm{T}}$. The computed flame lift-off height and its temporal variation agreed quite well with measured values. The influence of triple flame structure on the local propagation speed was considered in $G$-equation approach by Chen et al. [32] showing a favourable comparison of computed and measured [8, 42] lift-off heights. The jet velocity ranged from about 20 to $120 \mathrm{~m} / \mathrm{s}$ in the studies of Müller et al. [13] and Chen et al. [32]. Tabulated chemistry approach involving both premixed and non-premixed flamelets was also used in the past [43, 44] to study Sandia piloted jet diffusion flames.

In these flamelets based approaches, the random scalar mixing and reacting processes were treated to be statistically independent. This basically allowed the JPDF, $P(\zeta, \xi)$, to be written as a product of two marginal PDFs. The validity of this assumption was questioned using experimental [11, 27] and DNS [15, 45] data. Ruan et al. [46] retained this statistical dependency in their analysis and developed a mean reaction rate, $\overline{\dot{\omega}}$, model in the framework of Eq. (11) using both premixed and non-premixed flamelets. The JPDF was modelled using copula method proposed in [47] which allowed the statistical correlation between $Z$ and $c$ to be included in the analysis. The lift-off heights and flame-brush structure computed using this modelling approach agreed well with measured values for hydrogen jet lifted flames, which had exit velocities ranging from about 500 to $900 \mathrm{~m} / \mathrm{s}$. Elaborate detail on this modelling method and computational results can be found in [46].

It is well-known that lean combustion showing potentials for next generation of "green" combustion devices, is susceptible to instability issues leading to flashback and blow-off. These phenomena involve transient flame propagation char- 
acteristics which are akin to physical processes involved in transient evolution of lifted flames ignited at locations downstream of a jet exit. Studying this evolution of a lifted flame using unsteady RANS (URANS) approach is one of the aims of this study. It is rather challenging to quantitatively capture the transient flame propagation features using URANS for a range of dilution levels, jet velocities and spark positions. Although LES is ideally suited to capture these kind of transient phenomena, the current RANS work serves to test and understand the efficacies and limitations of partially premixed combustion model developed in [46] for URANS before attempting LES with this combustion modelling.

The specific objectives of this investigation are as follows. The first objective is to assess the ability of partially premixed combustion modelling in [46] to capture the lift-off heights for low jet velocities ranging from about 12 to $30 \mathrm{~m} / \mathrm{s}$ since it has been shown to perform well for jet velocities ranging from 500 to $900 \mathrm{~m} / \mathrm{s}$. The second objective is to study the effects of air dilution on the flame lift-off height using this combustion modelling. The third objective is to assess the efficacies of this combustion model for transient evolution of a lifted flame ignited at a downstream position as noted above. These objectives are addressed by comparing URANS simulation results to the measurements reported in [48].

This paper is organised as follows. The modelling framework and methodology are presented briefly in section 2 as elaborate detail can be found in [46]. The experimental test cases [48] used for this study is described in section 3. The numerical method and, boundary and initial conditions used for simulations are described in section 4 . The results are discussed in section 5 and the conclusions are summarised in the final section. 


\section{Modelling Methodology}

\subsection{Governing equations}

The Favre-averaged conservation equations for mass, momentum and total enthalpy, $\tilde{h}$, are solved. The two-equation $k-\epsilon$ model with modified constants as detailed in section 5.1 is used for turbulence because it is simple and adequate for jet flows considered in this study.

The approach described in [46, 47] is followed here to model the partially premixed combustion. The Favre-averaged transport equations for the first two moments of mixture fraction, $\widetilde{Z}$ and $\widetilde{Z^{\prime \prime 2}}$, and a reaction progress variable, $\widetilde{c}$ and $\widetilde{c^{\prime \prime 2}}$, are solved in addition to the above conservation equations. These four equations help us to characterise the scalar mixing and reaction progress at every point in the flow. Their mutual dependence is characterised and included in the modelling using the covariance, $\widetilde{Z^{\prime \prime} c^{\prime \prime}}$. These additional equations, in common notations, are written as

$$
\begin{aligned}
& \frac{\partial \bar{\rho} \widetilde{Z}}{\partial t}+\frac{\partial \bar{\rho} \widetilde{U}_{k} \widetilde{Z}}{\partial x_{k}}=\frac{\partial}{\partial x_{k}}\left(\overline{\rho D \frac{\partial Z}{\partial x_{k}}}-\overline{\rho u_{k}^{\prime \prime} Z^{\prime \prime}}\right), \\
& \frac{\partial \bar{\rho} \widetilde{Z^{\prime \prime 2}}}{\partial t}+\frac{\partial \bar{\rho} \widetilde{U_{k}} \widetilde{Z^{\prime \prime 2}}}{\partial x_{k}}=\frac{\partial}{\partial x_{k}}\left(\overline{\rho D \frac{\partial Z^{\prime \prime 2}}{\partial x_{k}}}-\overline{\rho u_{k}^{\prime \prime} Z^{\prime \prime 2}}\right) \\
& -2 \bar{\rho} \widetilde{\chi}_{Z}-2 \overline{\rho u_{k}^{\prime \prime} Z^{\prime \prime}} \frac{\partial \widetilde{Z}}{\partial x_{k}}, \\
& \frac{\partial \bar{\rho} \widetilde{c}}{\partial t}+\frac{\partial \bar{\rho} \widetilde{U}_{k} \widetilde{c}}{\partial x_{k}}=\frac{\partial}{\partial x_{k}}\left(\overline{\rho D \frac{\partial c}{\partial x_{k}}}-\overline{\rho u_{k}^{\prime \prime} c^{\prime \prime}}\right)+\overline{\dot{\omega}_{c}^{*}} \\
& \frac{\partial \bar{\rho} \widetilde{c^{\prime \prime 2}}}{\partial t}+\frac{\partial \bar{\rho} \widetilde{U}_{k} \widetilde{c^{\prime \prime 2}}}{\partial x_{k}}=\frac{\partial}{\partial x_{k}}\left(\overline{\rho D \frac{\partial c^{\prime \prime 2}}{\partial x_{k}}}-\overline{\rho u_{k}^{\prime \prime} c^{\prime \prime 2}}\right)-2 \bar{\rho} \widetilde{\chi}_{c} \\
& -2 \overline{\rho u_{k}^{\prime \prime} c^{\prime \prime}} \frac{\partial \widetilde{c}}{\partial x_{k}}+2 \overline{c^{\prime \prime} \dot{\omega}_{c}^{* \prime \prime}},
\end{aligned}
$$


and

$$
\begin{aligned}
\frac{\partial \bar{\rho} \widetilde{Z^{\prime \prime} c^{\prime \prime}}}{\partial t}+\frac{\partial \bar{\rho} \widetilde{U}_{k} \widetilde{Z^{\prime \prime} c^{\prime \prime}}}{\partial x_{k}}= & \frac{\partial}{\partial x_{k}}\left(\overline{\rho D \frac{\partial Z^{\prime \prime} c^{\prime \prime}}{\partial x_{k}}}-\overline{\rho u_{k}^{\prime \prime} Z^{\prime \prime} c^{\prime \prime}}\right)-2 \bar{\rho} \widetilde{\chi}{ }_{c c} \\
& -\overline{\rho u_{k}^{\prime \prime} c^{\prime \prime}} \frac{\partial \widetilde{Z}}{\partial x_{k}}-\overline{\rho u_{k}^{\prime \prime} Z^{\prime \prime}} \frac{\partial \widetilde{c}}{\partial x_{k}}+\overline{Z^{\prime \prime} \dot{\omega}_{c}^{* \prime \prime}}
\end{aligned}
$$

where $D$ is the molecular diffusivity and the turbulent scalar fluxes are modelled using the gradient hypothesis, for example, $\overline{\rho u_{k}^{\prime \prime} Z^{\prime \prime}}=-\bar{\rho} D_{t}\left(\partial \widetilde{Z} / \partial x_{k}\right)$ with $D_{t}=$ $v_{t} /$ Sc being the turbulent diffusivity. The eddy viscosity is calculated as $v_{t}=$ $C_{\mu} \widetilde{k}^{2} / \widetilde{\epsilon}$ using the computed $\widetilde{k}$ and $\widetilde{\epsilon}$. The turbulent Schmidt number, Sc, is taken to be 0.7 for all of the above scalars and, it is 1.0 for $\widetilde{k}$ and 1.3 for $\widetilde{\epsilon}$. The values of $C_{\mu}$ and other turbulence model parameters used in this study are discussed in section 5.1

The symbols $\widetilde{\chi}_{Z}$ and $\widetilde{\chi}_{Z c}$ in Eqs. (3) and (6) are respectively the Favre-averaged scalar dissipation rate of the mixture fraction fluctuations and cross dissipation rate. Following earlier studies, these two dissipation rates are modelled as

$\bar{\rho} \widetilde{\chi}_{Z} \equiv \overline{\rho D}\left(\frac{\partial Z^{\prime \prime}}{\partial x_{k}} \frac{\partial Z^{\prime \prime}}{\partial x_{k}}\right) \simeq C_{d} \bar{\rho}\left(\frac{\widetilde{\varepsilon}}{\vec{k}}\right) \widetilde{Z^{\prime \prime 2}}$

and

$\bar{\rho} \widetilde{\chi}_{Z c} \equiv \overline{\rho D}\left(\frac{\partial c^{\prime \prime}}{\partial x_{k}} \frac{\partial Z^{\prime \prime}}{\partial x_{k}}\right) \simeq C_{Z c} \bar{\rho}\left(\frac{\widetilde{\varepsilon}}{\bar{k}}\right) \widetilde{Z^{\prime \prime} c^{\prime \prime}}$

where the coefficients $C_{d}$ and $C_{Z c}$ denote ratios of scalar to turbulence time scales and both of them are taken to be 1.0 for this study [21, 46, 49].

These simple algebraic model was shown to be inadequate [50, 51] for the scalar dissipation rate of progress variable, $\widetilde{\chi}_{c}$. Many models are proposed recently to overcome this and these are summarised in [52]. The model proposed by Kolla et al. [53] for premixed combustion subsequently modified [54] to include mixture 
fraction stratification effects is used in this study because of its simplicity and robustness. This model is written as

$\bar{\rho} \widetilde{\chi}_{c} \equiv \overline{\rho D}\left(\frac{\partial c^{\prime \prime}}{\partial x_{k}} \frac{\partial c^{\prime \prime}}{\partial x_{k}}\right) \simeq \frac{\bar{\rho}}{\beta^{\prime}}\left(\left[2 K_{c}^{*}-\tau C_{4}\right] \frac{S_{\mathrm{L}}^{0}}{\delta_{\mathrm{L}}^{0}}+C_{3} \frac{\widetilde{\varepsilon}}{\bar{k}}\right) \widetilde{c^{\prime \prime 2}}$,

where $\beta^{\prime}=6.7, C_{3}=1.5 \sqrt{\mathrm{Ka}} /(1+\sqrt{\mathrm{Ka}})$ and $C_{4}=1.1 /(1+\mathrm{Ka})^{0.4}$ are model

parameters. The Karlovitz number is defined as $\mathrm{Ka}=\left(\delta / S_{\mathrm{L}}^{0}\right) /(\sqrt{v / \widetilde{\epsilon}})$, where $v$ is the local kinematic viscosity. The Zeldovich thickness, $\delta, \tau, S_{\mathrm{L}}^{0}$ and $\delta_{\mathrm{L}}^{0}$ depend on the local mixture fraction value and these are obtained from unstrained planar laminar premixed flame calculation. The model parameter $K_{c}^{*}$ is also obtained from the laminar flame calculation and this parameter varies with $Z$ [53] for this study.

The other terms related to combustion reactions in Eqs. (4) to (6) requiring

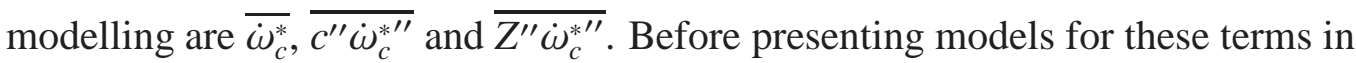
section 2.3, the definitions of $Z$ and $c$ used for this study are clarified next.

\subsection{Mixture fraction and progress variable}

Bilger's definition [55] of mixture fraction is followed here to describe the mixing between pure or air-diluted methane jet and air. This definition is written as

$Z \equiv \frac{2 Z_{\mathrm{C}} / W_{\mathrm{C}}+Y_{\mathrm{H}} / 2 W_{\mathrm{H}}+\left(Z_{\mathrm{O}, 2}-Z_{\mathrm{O}}\right) / W_{\mathrm{O}}}{2 Z_{\mathrm{C}, 1} / W_{\mathrm{C}}-Z_{\mathrm{O}, 1} / W_{\mathrm{O}}+Z_{\mathrm{H}, 1} / 2 W_{\mathrm{H}}+Z_{\mathrm{O}, 2} / W_{\mathrm{O}}}$

The mass fraction of an element $i$ and its atomic mass are denoted as $Z_{i}$ and $W_{i}$ respectively. Here, carbon, hydrogen and oxygen elements are used. The subscripts 1 and 2 denote the fuel and oxidiser streams respectively. Thus, $Z=1$ implies fuel jet regardless of its dilution and $Z=0$ implies the air stream. 
The progress variable indicating the progress of chemical reaction may be defined using different variables such as temperature, species mass fraction, etc. However, its specific definition depends on the problem of interest. Here, the sum of $\mathrm{CO}$ and $\mathrm{CO}_{2}$ mass fractions, $\psi=Y_{\mathrm{CO}}+Y_{\mathrm{CO} 2}$, is used for methane combustion and this definition is chosen because it allows a unique mapping of flamelet quantities with normalised progress variable [56], $c$, defined as

$c=\frac{\psi}{\psi^{\mathrm{Eq}}(Z)}$,

where $\psi^{\mathrm{Eq}}(Z)$ is the equilibrium value of $\psi$ for the local mixture fraction, $Z$, so that $c$ is bounded between 0 and 1. In principle, one can use $\psi$ as a progress variable instead of $c$ in Eq. (11). Here, the normalised form is chosen because it helps to clearly identify contributions originating from premixed and non-premixed modes to the mean reaction rate, $\overline{\dot{\omega}_{c}^{*}}$, as one shall see in the next subsection. Thus, one would be able to study the role of these individual contributions to the flame stabilisation mechanism and lift-off height.

\subsection{Reaction rate modelling}

Using the instantaneous transport equations for $Y_{\mathrm{CO}}$ and $Y_{\mathrm{CO} 2}$, it is straightforward to write a transport equation for instantaneous $c$ as has been done by Bray et al. [57]. The molecular mass diffusivities of $\mathrm{CO}$ and $\mathrm{CO}_{2}$ are taken to be the same as $D$, which is a reasonable approximation [58]. The apparent reaction rate, $\dot{\omega}_{c}^{*}$, in the instantaneous transport equation for $c$ can be written as [17, 29, 30, 57]

$\dot{\omega}_{c}^{*}=\frac{1}{\partial \psi / \partial c}\left(\dot{\omega}_{\psi}+2 \rho N_{Z c} \frac{\partial^{2} \psi}{\partial c \partial Z}+\rho N_{Z Z} \frac{\partial^{2} \psi}{\partial Z^{2}}+\rho N_{c c} \frac{\partial^{2} \psi}{\partial c^{2}}\right)$,

where $\dot{\omega}_{\psi}=\dot{\omega}_{\mathrm{CO}}+\dot{\omega}_{\mathrm{CO} 2}$ is the reaction rate for $\psi$. The three instantaneous scalar dissipation rates are defined as $N_{Z Z}=\rho D(\nabla Z \cdot \nabla Z), N_{Z c}=\rho D(\nabla c \cdot \nabla Z)$ and 
$N_{c c}=\rho D(\nabla c \cdot \nabla c)$. The derivatives in Eq. (12) become

$\frac{\partial \psi}{\partial Z}=c \frac{d \psi^{\mathrm{Eq}}}{d Z} \Rightarrow \frac{\partial^{2} \psi}{\partial Z^{2}}=c \frac{d^{2} \psi^{\mathrm{Eq}}}{d Z^{2}}$

$\frac{\partial \psi}{\partial c}=\psi^{\mathrm{Eq}} \Rightarrow \frac{\partial^{2} \psi}{\partial c^{2}}=0$, and $\frac{\partial^{2} \psi}{\partial Z \partial c}=\frac{d \psi^{\mathrm{Eq}}}{d Z}$

Substituting these derivatives into Eq. (12) and then averaging the resulting equation one obtains

$\overline{\dot{\omega}}_{c}^{*}=\overline{\dot{\omega}}_{c}+\underbrace{\rho N_{\mathrm{ZZ}} \frac{c}{\psi^{\mathrm{Eq}}} \frac{d^{2} \psi^{\mathrm{Eq}}}{d Z^{2}}}_{\bar{\omega}_{\mathrm{np}}}+\underbrace{2 \rho N_{Z c} \frac{1}{\psi^{\mathrm{Eq}}} \frac{d \psi^{\mathrm{Eq}}}{d Z}}_{\bar{\omega}_{\mathrm{cdr}}}$.

The first part signifies the contribution of premixed mode combustion, the second part, $\overline{\dot{\omega}}_{\text {np }}$, signifies the contributions from non-premixed mode and the third part, $\overline{\dot{\omega}}_{\text {cdr }}$, denotes a contribution resulting from interactions of $Z$ and $c$ gradients. Previous studies [29, 49] showed that the cross dissipation contribution is an order of magnitude smaller than the contributions from the other two terms and thus $\overline{\dot{\omega}}_{\text {cdr }}$ is neglected from further consideration in this work. The other two terms are modelled as follows.

The first term of Eq. (15) is modelled as [46]

$\overline{\dot{\omega}}_{c}=\bar{\rho} \int_{0}^{1} \int_{0}^{1}\left[\frac{\dot{\omega}_{c}(\zeta, \xi)}{\rho(\zeta, \xi)}\right] \widetilde{P}(\zeta, \xi) \quad d \zeta d \xi$,

where $\bar{\rho}$ is the mean local mixture density obtained as described in the later part of this subsection. The flamelet reaction rate, $\dot{\omega}_{c}(\zeta, \xi)$, and mixture density, $\rho(\zeta, \xi)$, are obtained from laminar unstrained premixed flame calculation. The Favre joint PDF, $\widetilde{P}(\zeta, \xi)$, including $Z$-c correlation is calculated using the copula method described in [46, 47]. This correlation is calculated using the covariance, $\widetilde{c^{\prime \prime}} Z^{\prime \prime}$, obtained from its transport equations, Eq. (6). 
Table 1: Model combination detail.

\begin{tabular}{lllll}
\hline Case & A & B & C & D \\
\hline Modelling of $\overline{\dot{\omega}}_{c}^{*}$ & $\overline{\dot{\omega}}_{c}$ & $\overline{\dot{\omega}}_{c}$ & $\overline{\dot{\omega}}_{c}+\overline{\dot{\omega}}_{\text {np }}$ & $\overline{\dot{\omega}}_{c}+\overline{\dot{\omega}}_{\mathrm{np}}$ \\
$\begin{array}{l}\text { Z-c correlation } \\
\text { included or not? }\end{array}$ & No & Yes & No & Yes \\
\hline
\end{tabular}

The second term, $\overline{\dot{\omega}}_{\text {np }}$, denoting contributions of non-premixed mode combustion is modelled as [46]

$\overline{\dot{\omega}}_{\mathrm{np}} \simeq \bar{\rho} \widetilde{c} \widetilde{\chi}_{\mathrm{Z}} \int_{0}^{1} \frac{1}{\psi^{\mathrm{Eq}}(\xi)} \frac{d^{2} \psi^{\mathrm{Eq}}(\xi)}{d Z^{2}} \widetilde{P}_{\beta}(\xi) d \xi$

These separate models and their modularity allow one to systematically study their individual influences on the lift-off height by including one effect at a time. This consideration yields four possible combinations of these models as listed in Table 1. The case A has contribution from only premixed mode without the effects of Z-c correlation, ie., the JPDF in Eq. (16) is modelled as the product of two marginal PDFs. The effect of this correlation is included in the case B. The cases $\mathrm{C}$ and $\mathrm{D}$ include contributions from premixed and non-premixed modes, and case $\mathrm{C}$ excludes the influences of $Z$ - $c$ correlation whereas case $\mathrm{D}$ includes this effect.

Strictly, one must include the contributions of three scalar dissipation rates in Eq. (12) at the flamelet level to close $\overline{c^{\prime \prime} \dot{\omega}_{c}^{* \prime \prime}}$ and $\overline{Z^{\prime \prime} \dot{\omega}_{c}^{* \prime \prime}}$ in Eqs. (5) and (6) respectively. This would need a multi-dimensional (in physical space) flamelet or alternatively the multidimensional flamelet generated manifolds with the three dissipation rates as controlling parameters [59, 60]. This adds further complexity into the modelling and so the approximations $\overline{c^{\prime \prime} \dot{\omega}_{c}^{* \prime \prime}} \approx \overline{c^{\prime \prime} \dot{\omega}_{c}^{\prime \prime}}$ and $\overline{Z^{\prime \prime} \dot{\omega}_{c}^{* \prime \prime}} \approx \overline{Z^{\prime \prime} \dot{\omega}_{c}^{\prime \prime}}$ 
are made here for the sake of simplicity. The validity of this approximation can be adjudged using comparisons with experimental measurements to be discussed in later part of this paper. The closure models for the above two terms are then written as [46, 47]:

$\overline{c^{\prime \prime} \dot{\omega}_{c}^{\prime \prime}} \approx \bar{\rho} \int_{0}^{1} \int_{0}^{1}(\zeta-\widetilde{\zeta}) \frac{\dot{\omega}_{c}(\xi, \zeta)}{\rho(\xi, \zeta)} \widetilde{P}(\xi, \zeta) d \xi d \zeta$

$\overline{Z^{\prime \prime} \dot{\omega}_{c}^{\prime \prime}} \approx \bar{\rho} \int_{0}^{1} \int_{0}^{1}(\xi-\widetilde{\xi}) \frac{\dot{\omega}_{c}(\xi, \zeta)}{\rho(\xi, \zeta)} \widetilde{P}(\xi, \zeta) d \xi d \zeta$

The temperature, $\widetilde{T}$, is calculated using the total enthalpy $\widetilde{h}$ computed in the simulation using its transport equation. This enthalpy includes the sensible and chemical parts as

$\widetilde{h}=c_{p, \text { mix }}\left(\widetilde{T}-T_{0}\right)+\Delta h_{f, \text { mix }}^{0}$,

where $T_{0}=298 \mathrm{~K}$ is a reference temperature. The mixture averaged specific heat capacity $c_{p, \text { mix }}$ and the enthalpy of formation $\Delta h_{f, \text { mix }}^{0}$ are calculated as

$c_{p, \operatorname{mix}}=\int_{0}^{1} \int_{0}^{1} c_{p}^{e}(\xi, \zeta) \widetilde{P}(\xi, \zeta) d \zeta d \xi$

$\Delta h_{f, \text { mix }}^{0}=\sum \int_{0}^{1} \int_{0}^{1} Y_{i} \Delta h_{f, i}^{0} \widetilde{P}(\xi, \zeta) d \zeta d \xi$,

The $c_{p, \text { mix }}$ given in Eq. (20) includes its temperature dependence through Eq. (21) while simulating turbulent combustion. An effective specific heat capacity, defined as $c_{p}^{e}=\left(\int_{T_{0}}^{T_{1}} c_{p} d T\right) /\left(T_{1}-T_{0}\right)$ is used to include the temperature dependence at the flamelet level and $T_{1}$ is the local temperature at which $c_{p}^{e}$ is calculated. The mixture molecular weight $W_{\text {mix }}$ required for the state equation is calculated using $W_{\text {mix }}=\int_{0}^{1} \int_{0}^{1}\left(\sum_{i} \frac{Y_{i}}{W_{i}}\right)^{-1} \widetilde{P}(\xi, \zeta) d \zeta d \xi$. 
The mean density is obtained using the ideal gas equation of state, $\bar{\rho}=\bar{p} W_{\text {mix }} / \widetilde{T} R_{0}$, with $\bar{p}$ being the thermodynamic pressure obtained from the simulation and $R_{0}=$ 8314.5 J/kmol-K is the universal gas constant. The Favre averaged scalar mass fractions are obtained using an integral equation similar to Eq. (21) and detailed description of these procedures are given in [46].

The various sources and sinks related to combustion can be precomputed and stored as a lookup table for turbulent flame simulation. This flamelet-table can be constructed using an arbitrarily complex chemistry and the GRI-Mech 3.0 involving 53 species and 325 reactions is used for the methane flames considered for this study. This table has five control parameters [46, 47], $\widetilde{Z}, \widetilde{c}, \widetilde{g}_{Z} \equiv \widetilde{Z^{\prime \prime 2}} /(\widetilde{Z}(1-\widetilde{Z}))$, $\widetilde{g}_{c}$ and $\widetilde{g}_{Z c} \equiv \widetilde{Z^{\prime \prime} c^{\prime \prime}} / \sqrt{\widetilde{Z^{\prime \prime 2}} c^{\prime \prime 2}}$. The number of points used in the construction of this table are respectively $24,21,21,21$ and 11 for this study. The numerical resolution of the table is finer around $\widetilde{Z}_{\mathrm{st}}$ and $\widetilde{c}=0.6$ because of large reaction rate near these locations. For turbulent flame simulations described in section 4 , these tabulated values are interpolated using a five-dimensional linear interpolation to get the various sources and sinks required for a spatial grid point and the error in this interpolation procedure was assessed to be about 1\% [46]. These techniques and models are used to simulate experimental test cases described next.

\section{Experimental test case}

The lifted flames established in the downstream of a methane jet into stagnant air were studied experimentally by Ahmed and Mastorakos [48]. These flames and their unsteady evolution from the initial spark location are used as test cases for this study. The burner consisted of an injection tube with an inner diameter of $d_{\mathrm{j}}=5 \mathrm{~mm}$ and a length of $128 d_{\mathrm{j}}$ to ensure a fully developed turbulent flow 
at the jet exit. There were no turbulence generating devices inside the fuel nozzle and the turbulence in the downstream of the nozzle exit is shear driven. In order to eliminate the disturbances from the room air currents, the fuel tube was surrounded by a coaxial laminar airflow having a diameter of $200 \mathrm{~mm}$ and a velocity of $0.1 \mathrm{~m} / \mathrm{s}$ [48]. The bulk mean velocity, $U_{\mathrm{j}}$, at fuel-jet exit ranged from 9 to $30 \mathrm{~m} / \mathrm{s}$ with corresponding Reynolds numbers of 2938 to 9793 . The influence of air dilution of the fuel jet on flame lift-off height was examined in the experiments [48] for four different dilution levels and the mole fractions of dilution air in the fuel jet considered were $X=10 \%, 20 \%, 30 \%$ and $40 \%$. A dilution level of $50 \%$ or more was reported to produce highly unstable flame and thus it was excluded in the experiments. The transient evolution of flame position from its initial sparking location was reported for $X=30 \%$ case. These flames are denoted as F0 (0\% dilution), F1, F2, F3 and F4 in this study. Since the behaviour of F0 and F1 were very similar, three flames, F0, F2 and F4 listed in Table 2 are considered for steady RANS simulations. Following the experiment, the F3 flame is used for URANS to study the transient flame propagation.

In all of these cases, the flame was ignited using an electrical spark at a far downstream, about 30 and $40 d_{\mathrm{j}}$, axial position. Different radial positions were also considered for experiments in [48] and the flame lift-off height was shown to be insensitive to the radial position of the spark for a given streamwise location. Thus, the sparks are located only along the centreline at different distances from the jet exit for this numerical work. The above two axial locations, 30 and $40 d_{\mathrm{j}}$, are considered to study the process of flame kernel growth, propagation, and its final stabilisation height. Both high-speed movies and OH PLIF (planar laser-induced fluorescence) imaging were used to visualise the flame propagation stages. The 
temporal variation of flame lift-off height and its final steady state value were analysed using direct high speed digital movies and OH-PLIF. The lift-off heights obtained by averaging 10 line-of-sight images for each instant during the evolution and various flame conditions were reported in [48]. The maximum variation of flame position at about the same elapsed time from ignition was measured to be about $9 \%$ using the limited sample size from the experiments.

\section{Numerical Setup}

A schematic of the numerical setup of the experiment is shown in Fig. 1. Because of axisymmetric nature of averaged flow and flame, the computational domains used for RANS and URANS simulations of this study are two-dimensional with boundary conditions marked in Fig. 11a. This domain extends to $300 d_{\mathrm{j}}$ in the streamwise, $z$, and $100 d_{\mathrm{j}}$ in the radial, $r$, directions. The computational domain is discretised using unstructured grid with fine mesh near the jet exit to resolve large spatial gradients in the near field of the fuel jet. A typical grid used for simulations is shown in Fig. 1b and it consists of 76,648 cells with the smallest size of $0.5 \mathrm{~mm}\left(0.1 d_{\mathrm{j}}\right)$. This grid is referred to as the base grid in the discussion below. A grid sensitivity study has been conducted with a coarser grid having the smallest cell size to be about $1 \mathrm{~mm}$ and a refined grid with $0.2 \mathrm{~mm}$ for the smallest cell size for F0 flame with $U_{\mathrm{j}}=16 \mathrm{~m} / \mathrm{s}$. The coarser grid was found to be inadequate to resolve flame brush structure, whereas the refined grid resulted in insignificant changes in velocity and mixture fraction variations compared to the standard grid. This test was repeated for F0 flame with $U_{\mathrm{j}}=30 \mathrm{~m} / \mathrm{s}$ and similar observations were made. Since the flame brush is expected to be thicker for air-diluted jet cases, the base grid was found to be adequate for other cases. 
The mass and momentum conservation equations are solved using the default setup in Fluent 14.0.0 package. The turbulence is modelled using the standard $k-\epsilon$ model in Fluent. The sources resulting from the pressure related terms are included using user defined functions (UDFs) as discussed in [46]. The scalar transport equations and combustion modelling equations are implemented in Fluent using user defined scalars (UDSs) and UDFs as described in [46] and thus the default combustion modelling in Fluent is completely circumvented. A transport equation for $\widetilde{h}$ and those given in Eqs. (2) to (6) are solved using UDSs. The modelling of various sources and sinks of these equations discussed in section 2 are included through UDFs. The fluid density is obtained using an user defined function involving $\widetilde{T}$ calculated from $\widetilde{h}$ as described in section 2.3 . The sources and sinks related to chemical reactions are obtained using the look-up table approach discussed earlier in section 2.3 . These calculation methodologies are described in detail by Ruan et al. [46].

\subsection{Boundary and initial conditions}

At the jet exit, which is the inlet boundary for computations, the mean streamwise velocity is specified using the $1 / 7$ th power law for a fully developed turbulent flow. The turbulent velocity fluctuation was estimated using a correlation involving a Reynolds number, Re, based on bulk-mean velocity and pipe diameter for a fully developed turbulent pipe flow. This correlation is given by $I \equiv u^{\prime} / U_{\mathrm{j}}=0.16 \mathrm{Re}^{-1 / 8}[61]$. The turbulence integral length scale at the jet exit is approximated to be about $0.7 d_{\mathrm{j}}$. A laminar flow with a velocity of $0.1 \mathrm{~m} / \mathrm{s}$ is used for the co-flowing air entering the computational domain as in Fig. 19. This same laminar flow is used for the entrainment boundary also. An adiabatic no-slip wall condition is used for the sidewall shown in Fig. 11a. 
For the scalar fields, $\widetilde{Z}$ is 1 at the jet exit and 0 for the air co-flow and the entrainment boundary. The values of $\widetilde{h}$ obtained using species enthalpies and their mole fractions are specified for the respective inlet streams. The other scalars, $\widetilde{c}$, $\widetilde{Z^{\prime \prime 2}}, \widetilde{c^{\prime \prime 2}}$ and $\widetilde{Z^{\prime \prime} c^{\prime \prime}}$ are set to be zero at the inlet boundaries. For the sidewall, the normal gradient of these scalars are specified to be zero.

\subsection{Flame kernel initialisation}

The flame is ignited numerically on a fully converged cold flow and scalar mixing solution. Following the experiments in [48], the flame kernel is initialised on the jet axis at a downstream location of about 30 and $40 d_{\mathrm{j}}$ from the jet exit. This kernel has a size of $4 \times 4 \mathrm{~mm}^{2}$ and it is set to have $\widetilde{c}=1$ representing fully burnt products. The energy in this kernel is about $100 \mathrm{~mJ}$ as provided by a spark in the experiments of [48]. Different sizes and energy levels of this initial kernel are tested and it is found that the final lift-off height is not influenced by these parameters. Detailed modelling of the spark ignition and its plasma is beyond the scope of this paper. Some attempt in this regard was made by Lacaze et al. [40] using one-step chemistry and an energy deposition ignition model in their LES study. However, only one case having $X=30 \%$ and $U_{\mathrm{j}}=25.5 \mathrm{~m} / \mathrm{s}$ was considered in [40] because of high computational cost for LES. All of the RANS simulations reported in this study are started by initialising a kernel as described above in the respective converged cold flow and scalar mixing solutions.

\section{Results and Discussion}

\subsection{Cold-flow validation}

The turbulence models, their parameters, and boundary conditions used in this study are validated first by simulating a cold-jet with an exit velocity of 
$U_{\mathrm{j}}=21 \mathrm{~m} / \mathrm{s}$ corresponding to $\operatorname{Re}=6800$. The jet fluid is air as used in the experiments of [48]. The model parameters of the standard $k-\epsilon$ equations are chosen to be $C_{\mu}=0.065, C_{\epsilon 1}=1.44$ and $C_{\epsilon 2}=1.85$ based on an earlier study [62]. Figure 2 compares the radial variation of computed and measured streamwise, $z$, velocity and its r.m.s. (root mean square) values for five $\widehat{z}=z / d_{\mathrm{j}}$ locations. The mean velocity is scaled as $\mathcal{U}=\left(\widetilde{U}-U_{\mathrm{c}}\right) /\left(\widetilde{U}_{\mathrm{cl}}-U_{\mathrm{c}}\right)$, where $U_{\mathrm{c}}=0.1 \mathrm{~m} / \mathrm{s}$ is the co-flow velocity and $\widetilde{U}_{\mathrm{cl}}$ is the centreline value at the respective $\widehat{z}$ location. The r.m.s. value is scaled as $\mathcal{U}_{\mathrm{rms}}=u_{\mathrm{rms}} /\left(\widetilde{U}_{\mathrm{cl}}-U_{\mathrm{c}}\right)$ and $u_{\mathrm{rms}}=\sqrt{2 \widetilde{k} / 3}$ using the computed values of $\widetilde{k}$. The symbols in Fig. 2 are measured values reported in [48] and the lines are computational results. The agreement seen in this figure is excellent and supports the self-similar behaviour of the jet. The computed axial variation of centreline velocity scaled as $\mathcal{U}_{\mathrm{cl}}=\left(\widetilde{U}_{\mathrm{cl}}-U_{\mathrm{c}}\right) /\left(U_{\mathrm{j}}-U_{\mathrm{c}}\right)$ agrees quite well with the empirical relationship given in [63] as shown in Fig. 3. This lends further support for the self-similar behaviour of the computed turbulent jets. The comparisons shown in Figs. 2 and 3 are very good. These results also support the values used for turbulence model parameters and thus they are adopted for combustion simulations reported here.

Unfortunately, no measurements were reported for the mixture fraction field in [48], however, it was argued that the mean mixture fraction, $\widetilde{Z}$, variation in these open jets can be approximated well using a correlation given by [64]

$\widetilde{Z}(z, r)=9.52 \sqrt{\frac{\rho_{\mathrm{j}}}{\rho_{\text {air }}}}\left(\frac{d_{\mathrm{j}}}{2 z-7.2 d_{\mathrm{j}}}\right) \exp \left[-59\left(\frac{r}{z-3.6 d_{\mathrm{j}}}\right)^{2}\right]$,

where $\rho_{\mathrm{j}}$ is the density of the jet fluid. The mixture fraction can be related to the equivalence ratio using $\phi=\widetilde{Z}\left(1-\widetilde{Z}_{\mathrm{st}}\right) /\left(\widetilde{Z}_{\mathrm{st}}(1-\widetilde{Z})\right)$. A typical comparison of $\phi$ contours obtained using the above correlation and the computational result for flame F3 having $U_{\mathrm{j}}=25.5 \mathrm{~m} / \mathrm{s}$ and $U_{\mathrm{c}}=0.1 \mathrm{~m} / \mathrm{s}$ is shown in Fig. 4, The three 
contours shown in Fig. 4 correspond to the stoichiometry, lean and rich flammability limits for methane-air mixture at $300 \mathrm{~K}$. The good comparison shown here is similar to that observed in [40] and it further supports the turbulence and scalar mixing models used for this study.

The combustion simulations are started using converged cold-flow solutions with sparking at two axial locations as described in subsection 4.2. All the flames, except F3, are computed using RANS approach since the stabilised flame lift-off height is of main interest. The flame F3 is simulated using URANS approach to study the unsteady flame evolution as experimental data are available for this flame. In the followings, the RANS results are discussed first to address the first two objectives of this study before presenting the transient evolution of F3 flamebrush from its initial sparking location, which is related to the third objective of this investigation.

\subsection{Flame lift-off height}

The modularity of premixed and non-premixed combustion models and Z-c correlation allows four different model combinations to assess their individual effects and mutual dependencies, as noted in section 2.3. The results of this assessment on the flame lift-off height for the four cases listed in Table 1 are shown in this subsection. It is worth to remind ourselves here that case A has contribution only from premixed mode combustion and case B includes Z-c correlation effect along with premixed combustion through JPDF in Eq. (16). The other two cases

include both the non-premixed and premixed combustion contributions, but with Z-c correlation effect excluded in case $\mathrm{C}$ and included in case D.

Figure 5 shows the computed mean temperature field and mixture fraction contours for F0 (undiluted) and F4 (highly diluted) flames having the same jet 
velocity, $U_{\mathrm{j}}=16 \mathrm{~m} / \mathrm{s}$. Note that the two flames are plotted in two different scales, and the radial and axial distances are normalised using $d_{\mathrm{j}}$. The $\widetilde{Z}_{\mathrm{st}}$ contour (as a thick line) and lift-off height $\left(L_{\mathrm{f}}\right)$ are highlighted in this figure. The flame liftoff height is the most upstream point of $\widetilde{T}=1200 \mathrm{~K}$ contour which corresponds to $5 \%$ of the maximum $\mathrm{OH}$ concentration as used in the experiment [9]. The other two iso-lines (thin black lines) of Favre-averaged $Z$ correspond to the lean and rich flammability limits of methane ( $\phi=0.5$ and 1.5 respectively). It is shown that the highest temperature at the flame leading edge is close to the Favreaveraged stoichiometric mixture fraction, $\widetilde{Z}_{\mathrm{st}}$, for both F0 and F4 flames as one would expect. The maximum temperature is found at further downstream where the $\widetilde{Z}_{\mathrm{st}}$ contour intersects with the jet centre axis, approximately at about 130 to $150 d_{\mathrm{j}}$ for flame $\mathrm{F} 0$; whereas for the diluted flame $\mathrm{F} 4$, it falls in between $70 d_{\mathrm{j}}$ and $90 d_{\mathrm{j}}$ because this intersection point is located more upstream due to the air dilution in the jet fluid.

Table 2 compares the computed $L_{\mathrm{f}} / d_{\mathrm{j}}$ of flames F0, F2 and F4 for the four cases listed in Table 1 . The jet velocity is $16 \mathrm{~m} / \mathrm{s}$. The pure premixed case A excluding the $Z$ - $c$ correlation gives $L_{\mathrm{f}} / d_{\mathrm{j}} \approx 5$ and 5.5 for F0 and $\mathrm{F} 2$ respectively, lower than the measured values of 5.8 and 6.7. For the highly diluted $(X=40 \%)$ flame F4, case A overestimates the lift-off height by about $2 d_{\mathrm{j}}$ as in Tables 2 . The modelling case B includes the $Z$-c correlation and yields slightly larger values of $L_{\mathrm{f}} / d_{\mathrm{j}}$ for F0 and F2, whereas for F4 a decrease in $L_{f} / d_{\mathrm{j}}$ is observed. The reason for this will be discussed later in section 5.2.2 while examining the mean reaction rate $\overline{\dot{\omega}}_{c}$. Case $\mathrm{C}$ includes contribution of non-premixed combustion and the lift-off height computed in this case is increased by about one diameter compared to the case A. This effect is further discussed in next section 5.2.1. Finally, when Z-c 
Table 2: Comparison of computed and measured [48] final lift-off heights, $L_{\mathrm{f}} / d_{\mathrm{j}}$, for $U_{\mathrm{j}}=16 \mathrm{~m} / \mathrm{s}$.

\begin{tabular}{llllllll}
\hline Flame & Air\% & $\widetilde{Z}_{\text {st }}$ & Exp. & A & B & C & D \\
\hline F0 & 0 & 0.055 & 5.5 & 4.9 & 5.3 & 5.4 & 5.6 \\
F2 & 20 & 0.08 & 6.7 & 5.5 & 5.6 & 5.7 & 5.9 \\
F4 & 40 & 0.12 & 16.3 & 18.4 & 13.7 & 18.8 & 16 \\
\hline
\end{tabular}

correlation and non-premixed combustion effect are both included in the modelling case $\mathrm{D}$, the computed lift-off heights are in excellent agreement with the experiments for F0 and F4 as seen in Table 2 for this case. The agreement is not as good for F2 but still within the $9 \%$ uncertainties noted in the experiments [48]. The modelling case $\mathrm{D}$ is chosen for further testing with different jet velocities and air-dilution levels in section 5.2.3.

\subsubsection{Role of non-premixed combustion mode}

The contributions of non-premixed combustion is given by Eq. (17), which involves the second derivative term, $d^{2} \psi^{\mathrm{Eq}} / d Z^{2}$. Thus, the contributions from $\overline{\dot{\omega}}_{\text {np }}$ are expected for mixtures within the flammability limits. The variations of $\psi^{\mathrm{Eq}}=Y_{\mathrm{CO}}^{\mathrm{Eq}}+Y_{\mathrm{CO}_{2}}^{\mathrm{Eq}}$ and its derivatives with $\left(Z-Z_{\mathrm{st}}\right)$ are shown in Fig. 6 for mixtures close to stoichiometry. The flammability limits correspond to about -0.027 and 0.037 in Fig. 6. Note that the derivatives are scaled appropriately to fit in the range of $y$-axis shown in this figure. As expected the second derivative peaks near the stoichiometric location and has a larger negative part than positive part. Hence, the overall contribution from $\overline{\dot{\omega}}_{\text {np }}$ to $\overline{\dot{\omega}}_{c}^{*}$ in Eq.(12) is negative. The diffusion effect of $c$ in $Z$ space, signified by $\partial^{2} \psi / \partial Z^{2}$, prevents the local chemical reactions to 
reach their equilibrium and thus decreases the flame propagation speed as noted by Bray et al. [17, 57]. This decrease in the flame propagation speed increases the lift-off height when non-premixed combustion contribution is included in the modelling, compare cases A \& C and cases B \& D in Table 2. This effect is observed for the range of velocities and dilution levels considered in this study.

To further understand the relative role of non-premixed combustion contributions to the overall mean reaction rate, $\overline{\dot{\omega}_{c}^{*}}$, the spatial variations of $\overline{\bar{\omega}_{c}^{*}}$ and its components, $\overline{\dot{\omega}}_{c}$ and $\overline{\dot{\omega}}_{\text {np }}$, are shown in Fig. 7 for flames F0 and F4. This result is shown for the modelling case $\mathrm{D}$ and for $U_{\mathrm{j}}=16 \mathrm{~m} / \mathrm{s}$. As one would expect, the significant reaction rates occur within the flammability limits and the peak rate is around the stoichiometric contour. The relative contribution of non-premixed mode varies significantly between the flames F0 and F4. Figures $7 \mathrm{~b}$ and $7 \mathrm{k}$ show that the magnitude of peak $\overline{\dot{\omega}}_{\text {np }}$ is about an order of magnitude lower than $\overline{\dot{\omega}}_{c}$ in the flame F0 and this difference becomes two orders of magnitude for the flame F4 as seen in Figs. 7 and 7f. This is because of the availability of oxygen in the jet fluid because of air-dilution. Despite the small contribution from non-premixed combustion mode, it is found to be important to obtain the correct lift-off height as noted in section 5.2. These observations on the localness of $\bar{\omega}_{\text {np }}$ supports the assumption of Müller et al. [13] to account for partially premixed combustion effects in $G$-equation approach. The relatively larger contribution of $\overline{\dot{\omega}}_{\text {np }}$ in the flame F0 compared to F4 results from larger values of $\widetilde{\chi}_{Z}$ because the flame F0 stabilises in regions relatively closer to the jet exit with larger shear and mixture fraction gradients. Thus, the oxygen transported by turbulence large-scale rollup leading to enhanced entrainment is likely to play an important role in the stabilisation of flame F0. In the flame F4, the effect of entrained oxygen is expected to be delayed 
downstream because of the presence of some oxygen in the fuel diluted with air. Thus, it is becoming imperative that the role of $Z-c$ correlation signifying the mutual influences of scalar mixing and chemical reactions need to be understood and this is discussed next. The influence of this correlation on the flame lift-off height is observed already in Fig. 5] and Table 2.

\subsubsection{Effect of $Z$-c correlation}

The effects of Z-c correlation on $\overline{\dot{\omega}}_{c}$, Eq. (16), are shown in Fig. 8 for the flames F0 and F4 having $U_{\mathrm{j}}=16 \mathrm{~m} / \mathrm{s}$. The values of $\overline{\dot{\omega}}_{c}$ computed using models $\mathrm{A}$ and $\mathrm{B}$ (see Table 1) are compared to understand the role of $Z$ - $c$ correlation. In these two models, the influences arising from non-premixed combustion are excluded by omitting $\overline{\dot{\omega}}_{\text {np }}$ given by Eq. (17) while calculating $\overline{\dot{\omega}}_{c}^{*}$. The computed covariance contours shown in Figs. $8 \mathrm{~b}$ and $8 \mathrm{~d}$ respectively for the flame F0 and F4 suggest that $\widetilde{Z^{\prime \prime} c^{\prime \prime}}$ changes its sign near $\widetilde{Z}_{\mathrm{st}}$ which is consistent with a previous DNS study [49]. This sign change is because, locally richer mixture (positive $\left.Z^{\prime \prime}\right)$ in the lean side can promote combustion resulting in enhanced reaction rate (positive $c^{\prime \prime}$ ). This gives $\widetilde{Z^{\prime \prime} c^{\prime \prime}}>0$ for the lean mixture. The locally richer mixture in the rich side can make the mixture difficult to burn reducing the overall reaction rate (negative $c^{\prime \prime}$ ). This yields $\widetilde{Z^{\prime \prime} c^{\prime \prime}}<0$ for the rich side as seen in Fig. 8. These results are consistent with an earlier analysis of turbulent stratified combustion [47].

Including the $Z-c$ correlation redistributes $\overline{\dot{\omega}}_{c}$ inside the flame brush as seen in Fig. 8. Two main effects can be observed by comparing Figs. $8 \mathrm{a}$ to $8 \mathrm{~b}$ for flame F0 and $8 \mathrm{c}$ to $8 \mathrm{~d}$ for flame F4. These two effects are as follows. (1) The region having high mean reaction rate near $\widetilde{Z}_{\mathrm{st}}$ becomes smaller when $Z$-c correlation is included in the analysis and this is prominent for the air-diluted flame F4. One 
could see a reduction in the reaction zone width near the lift-off height by about $d_{\mathrm{j}}$ for F4 by comparing Figs. $8 \mathrm{c}$ and $8 \mathrm{~d}$. (2) The two flame branches merge in downstream of the leading edge and the merged flame brush moves towards $\widetilde{Z}_{\text {st }}$ (radial squashing of the flame brush), which is more prominent for the flame F4. The first effect, a decrease in the reaction zone width, increases the lift-off height because of relatively weaker flame propagation along the $\widetilde{Z}_{\text {st }}$ contour. However, the second effect causing radial squashing of the flame brush moves the leading edge upstream.11 Thus, there is a fine balance between these two opposing effects at the leading edge for the flame brush to stabilise at the final lift-off height. The following is postulated here. The rich flame branch which is typically located in relatively high velocity region moves away from the jet centreline because of radial squashing. Thus, the flame brush leading edge supported by the downstream chemical activity experiences a lower velocity at this axial position. This enables the leading edge to propagate towards its final stabilisation region. Therefore, the overall effect of Z-c correlation can be seen as a combination of the above two effects and their predominant role can vary depending on the turbulence and thermo-chemical conditions.

The effects of $Z$-c correlation also changes the relative importance of non-

1 The upstream movement of the flame edge is because of the following reason. The total amount of heat released by burning a given amount of fuel must be conserved and this conservation can only be achieved by either an increase in the magnitude of the mean reaction rate or an extension of the flammable region in the axial direction when there is radial squashing of the flame brush because this squashing leads to a reduction of the flammable region in radial direction. The increase in the reaction rate magnitude is not observed in this study. Since the fuel is present only near the jet exit, the flame edge will have to move upstream to conserve the total heat release rate. 
premixed combustion mode contribution. In the highly diluted flame F4, as the premixed lean and rich reaction zones merge and move towards $\widetilde{Z}_{\mathrm{st}}$ contour where $\overline{\dot{\omega}}_{\text {np }}$ tends to be large the non-premixed combustion plays an important role for the overall mean reaction rate. This substantially influences the lift-off height as given in Table 2. The difference in $\left(L_{\mathrm{f}} / d_{\mathrm{j}}\right)$ is about 0.4 due to non-premixed combustion contribution (compare the values for $\mathrm{A}$ and $\mathrm{C}$ in Table 2) for flame $\mathrm{F} 4$, and this difference becomes 2.3 when $Z$ - $c$ correlation is included (compare B and $\mathrm{D}$ in Table 2 for F4). This supports the above observation on the role of $Z$ $c$ correlation to obtain lift-off heights measured in experiments. This correlation effect is observed to be small for the undiluted methane flame F0 (see values in Table 2) whereas this effect was observed to be significant for an undiluted hydrogen jet flame [46]. These observations suggest that the flammability limits of the fuel can alter the importance of $Z-c$ correlation effects - the effects are stronger when the flammability limit is wider. The level of partial premixing can also influence the $Z-c$ correlation effects. In the lifted jet flames, the partial premixing is affected by the entrainment influenced by the jet velocity and the dilution level. The influence of these two parameters on the flame lift-off height is discussed next.

\subsubsection{Influence of jet velocity and air-dilution}

To further assess and confirm the role of contributions from non-premixed combustion and Z-c correlation for other jet velocities, the flame F4 is computed using the four modelling cases, A to D in Table 1, for three different velocities. The flame lift-off heights obtained from these calculations are shown in Fig. 9a. The model A involving only the premixed combustion mode without Z-c correlation overestimates the lift-off height and including the correlation in model B 
leads to an underestimate. This underestimate seems to be significant for low jet velocity considered for this testing. When contributions of non-premixed combustion are included in model $\mathrm{C}$ then the overestimate of $L_{\mathrm{f}} / d_{\mathrm{j}}$ increases further by about 0.5 to 1 . Including the contributions of both non-premixed combustion and $Z$ - $c$ correlation gives $L_{\mathrm{f}} / d_{\mathrm{j}}$ values close to the measured values for all the jet velocities and flames investigated in this study, supporting the observations made in the previous subsections on the roles of these contributions. Thus, the model D is used for further investigation of this study to address the air-dilution effects and transient evolution.

The effect of air-dilution on $L_{\mathrm{f}} / d_{\mathrm{j}}$ is shown in Fig. $9 \mathrm{~b}$ by considering F0, F2 and F4 flames. The computed and measured lift-off heights of these flames are compared in this figures for a range of jet velocities used in the experiments of [48]. As one can see in this figure, this comparison is uniformly very good for all the cases. For a given jet velocity, $L_{\mathrm{f}} / d_{\mathrm{j}}$ increases with dilution level. This is because an increase in the stoichiometric mixture fraction value resulting from dilution moves $\widetilde{Z}_{\mathrm{st}}$ contour towards the jet centre where the local velocities are expected to be large. Thus, the diluted flame stabilises at a farther downstream location compared to the undiluted flame. For F4 flame, the highest jet velocity considered is $22 \mathrm{~m} / \mathrm{s}$ and a higher velocity leads to flame blow-off in the simulation, which is consistent with observation in the experiments [48]. Thus, it seems that the modelling frame work used in this study is able to capture the flame liftoff heights of a range of conditions, dilution levels, jet velocities ranging from 12 to $30 \mathrm{~m} / \mathrm{s}$ and 500 to $900 \mathrm{~m} / \mathrm{s}$ in [46] for undiluted hydrogen, without having to change the combustion modelling parameters. The reason for such robust and consistently good behaviour of the combustion model is because of close coupling of 
the model parameters to the underlying important physical processes controlling the local burning rate as discussed in [46, 65-68].

\subsubsection{Stabilisation mechanism}

Many theories [20, 22, 23] for flame stabilisation mechanism at the leading edge have been proposed in past studies and they include premixed flame propagation [3-5, 20], extinction of diffusion flamelets [1], triple flames [6, 14], the large-scale eddy model [2] as well as the edge flame concept [24]. Detailed discussion of these theories is not the main objective here but some remarks can be made based on the results obtained from the RANS simulations conducted in this study. The two classical theories based on premixed flame propagation and extinction of diffusion flamelets are discussed here.

Figure 10 shows the velocity field in the region of flame stabilisation for flames F0 and F4 computed using the model D in Table 1. The flame brush leading edge in these two flames is located in regions with low-velocity with a value of about $0.4 \mathrm{~m} / \mathrm{s}$ in the immediate upstream of the leading edge. This value is close to the planar laminar premixed flame speed for stoichiometric methane/air mixture. This phenomenon is consistent with many previous experimental [7, 69] and numeri-

cal [18] studies. Another notable point is that the main reaction zone with high heat release rate per unit volume is located relatively closer to the jet centre than the leading edge which is consistent with the experimental observation [11]. This is more evident in the highly diluted flame F4 depicting a strong flow divergence and streamline deflection as seen in Fig.10b.

Peters and Williams [1] suggested that the lifted flame stabilises due to extinction of diffusion flamelets at the leading edge resulting from high scalar dissipation rate. Although this concept was claimed to be inadequate [21, 27, 70, 71], 
the influence of flamelets extinction should not be overlooked in the stabilisation process as noted in [22] and may be responsible for the hysteresis phenomenon observed for the lift-off height [23]. The combustion model given by Eq. (15) supports this view since the scalar dissipation rate, $\widetilde{\chi}_{Z}$, plays a role for $\overline{\dot{\omega}_{c}^{*}}$ and thus on the turbulent flame propagation speed. Therefore, the normalised scalar dissipation rate, $\widetilde{\chi}_{Z} / \widetilde{\chi}_{Z, q}$, in the flame stabilisation region computed using the model D is shown in Fig. 11 along with the mean reaction rate field. A typical extinction value of $5 \mathrm{~s}^{-1}$ for methane-air flame is used for $\widetilde{\chi}_{Z, q}$ based on earlier studies [18, 21]. It is observed that the normalised $\widetilde{\chi}_{Z}$ values in the stabilisation region are significantly smaller than 1, approximately $5 \%$ and $0.5 \%$ for F0 and F4 flames respectively. This considerably smaller value of $\widetilde{\chi}_{Z} / \widetilde{\chi}_{Z, q}$ at the leading edge of the air-diluted flame is because of the smaller mixture fraction gradient in the flame F4 resulting from air-dilution. It is to be noted that $U_{\mathrm{j}}=16 \mathrm{~m} / \mathrm{s}$ is the same for both F0 and F4 flames shown in Figs. 10 and 11. This suggests that the role of non-premixed flamelets extinction on flame stabilisation is relatively more important for undiluted flames compared to diluted flames. This is reflected in the $L_{\mathrm{f}} / d_{\mathrm{j}}$ values listed in Table 2. Including the non-premixed combustion effects in the model $\mathrm{C}$ moves the flame brush leading downstream by about $10 \%$ compared to the case A catering only for premixed flamelets for the flame F0. This difference is only about $2 \%$ for the flame F4 as listed in Table 2 . Thus, the role of contributions from premixed and non-premixed combustion must be included in the modelling of turbulent partially premixed flames. The influence of large-scale turbulence on the flow and scalar mixing is included inherently by solving the transport equations for the Favre averaged momentum and scalar mass fraction conservations. Thus, the modelling framework used in this work seem to have the ability to in- 
clude the relevant important physical processes. Also, the presence of triple and edge flame structures in an averaged sense is also captured well by this modelling as has been evidenced in the mean reaction rate fields shown in Figs. 7, 8, 9, 10 and 13. The unsteady evolution is shown in Fig.13, which is to be discussed next.

\subsection{Temporal evolution of lift-off height}

The temporal evolution of flame F3 from its initial ignition location to its final stabilisation region was measured in the experiment of [48] using high-speed digital movies. The temporal variation of axial position of the most upstream flame edged detected from the 10 recorded movies was averaged in a consistent manner using frame by frame. This averaged flame position was then plotted as a function of elapsed time from spark initiation. Four cases in total, two jet velocities of 12.5 and $25.5 \mathrm{~m} / \mathrm{s}$, and two ignition locations of 30 and 40 fuel jet diameters, are studied. The experimental results for these four cases are shown in Fig. 12] The error bar corresponds to $9 \%$ error reported in the experimental study using the limited samples (10 frames for a given time). The URANS computational results are obtained using the model $\mathrm{D}$ in Table 1 and a time step of $5 \times 10^{-5} \mathrm{~s}$, which is smaller than the laminar flame timescale defined as $\tau_{\mathrm{L}} \equiv\left(\delta_{\mathrm{L}} / S_{\mathrm{L}}\right) \simeq 1.2 \times 10^{-3} \mathrm{~s}$ for stoichiometric $\mathrm{CH}_{4}$-air mixture. The unsteady simulations are performed until a stable liftoff height is obtained.

The time axis in Fig. 12] is normalised using both a jet flow timescale defined as $\left(d_{\mathrm{j}} / U_{\mathrm{j}}\right)$ and $\tau_{\mathrm{L}}$. The time normalised using $\tau_{\mathrm{L}}$ is shown on the top. The most leading edge of the flame is identified using $\widetilde{T}=1200 \mathrm{~K}$ as noted in section 5.2 , After the ignition, the flame first moves downstream slightly due to the local flow convection in the experiments and this process is captured well in the computations, which is more apparent for $U_{\mathrm{j}}=25.5 \mathrm{~m} / \mathrm{s}$ case shown in Fig. 12h. The flame 
then propagates towards its final stabilisation region against the flow. The convergence of flame tracks for two different ignition locations suggests that the final lift-off height does not depend on the initial spark location for both $U_{\mathrm{j}}=25.5$ and $12.5 \mathrm{~m} / \mathrm{s}$ cases shown respectively in Figs. $12 \mathrm{l}$ and $12 \mathrm{~b}$. Comparing these two figures also identifies that the flame takes longer to reach its final stabilisation height in the higher jet velocity case. The results in these figures show that it takes $t^{+} \sim 300$ for the influences of the initial transients arising from the spark location to become negligible irrespective $U_{\mathrm{j}}$ values (see the gap between the computational curves for a given $U_{\mathrm{j}}$ ). Also, the final lift-off height is reached within a few percent by $t^{+} \sim 300$ for $U_{\mathrm{j}}=12.5 \mathrm{~m} / \mathrm{s}$ as seen in Fig. 12 $\mathrm{b}$. This normalised time becomes about 700 for $U_{\mathrm{j}}=25.5 \mathrm{~m} / \mathrm{s}$ suggesting that the time taken to reach the final lift-off height is proportional to the jet velocity when these are normalised using the stoichiometric flame scales, i.e., $t_{\text {final }}^{+} \sim U_{\mathrm{j}}^{+}$, where $U_{\mathrm{j}}^{+}$ is $U_{\mathrm{j}} / S_{\mathrm{L}}$. This scaling suggests that the premixed flame propagation plays a vital role in the establishment of lifted flame from an initial kernel. There are some differences between the experimental and computational results but it should be noted that these are URANS calculations and the sample size available for the experimental analysis was limited. Nevertheless, the trends and important features observed in the experiments for transients are captured reasonably well by the URANS calculations.

Furthermore, the slopes of computed $z / d_{\mathrm{j}}$ vs $t^{+}$curves corresponding to the flame brush propagation speed agree quite well with the experimental values for the flames ignited at $z=30 d_{\mathrm{j}}$ for both jet velocities shown in Fig. 12, A considerable difference is seen for flames ignited at $z=40 d_{\mathrm{j}}$. The possible reasons for this are as follows: (1) the experimental data size is limited (10 samples) and thus 
the averaged $z / d_{\mathrm{j}}$ values may have some statistical bias and more data is needed to obtain a better average and (2) more importantly, the dynamic interaction between large-scale flow structures and flame propagation is limited in RANS methodology and this requires a more advanced approach such as LES.

\subsubsection{Stages of flame-brush propagation}

Figure 13 shows contours of $\overline{\bar{\omega}_{c}^{*}}$ using pseudo colours at five different times noted in the figure. These times are chosen to highlight the important stages of the flame brush evolution. The results shown in Fig. 13 are typical for this evolution and it shown for $U_{\mathrm{j}}=25.5 \mathrm{~m} / \mathrm{s}$ with spark initiation at $z / d_{\mathrm{j}}=30$, the case shown in Fig. 12a. The contours of $\widetilde{Z}_{\text {st }}$, lean and rich flammability limits are also shown as lines. The various stages of flame evolution discussed below compares very well with the experimental observations using high-speed movies in [48].

\section{Downstream convection:}

The flame kernel initialised at $z / d_{\mathrm{j}}=30$ is first convected downstream very quickly by the mean flow as seen in the first frame of Fig. 13. In this early stage of flame development, the kernel growth is mainly due to both molecular and turbulent diffusion of deposited energy and thus it retains a spherical shape as seen in Fig. 13 for $t^{*}=5$. This spherical evolution transitions into a second phase.

\section{Radial expansion and downstream propagation:}

As the flame kernel moves into more flammable mixture bounded between $\widetilde{Z}_{\mathrm{r}}$ and $\widetilde{Z}_{\mathrm{st}}$, the flame starts to propagate in the radial direction under the influence of streamwise convection by the mean flow. This results in the reaction zone shape as seen in the second frame of Fig. 13] shown for $t^{*}=30$. 
The flame brush upstream edge remains at about the same position during this stage. From this second stage, the flame brush transitions into a third stage involving edge flame propagation.

3. Upstream propagation and stabilisation:

During the transition from the second stage, the flame brush positions itself between the rich and lean flammability limits (in an average sense) with stronger reactions occurring near the stoichiometry. This gives a typical triple flame structure as shown in the third frame of Fig. 13 for $t^{*}=138$. By this time, the total width of the flame brush reaches about $7 d_{\mathrm{j}}$ in the radial direction as in Fig. 13. Once the flame brush attains this typical shape, then it starts to propagate upstream as an edge flame. This propagation is led along $\widetilde{Z}_{\mathrm{st}}$ contour until the final stabilisation height is reached as shown in the last frame of Fig. 13. A similar observation was also made by Müller et al. [13] using a different modelling approach involving $G$ equation.

These three stages of flame brush evolution are clearly seen when the kernel is initialised in a mixture close to the rich flammability limit. When the kernel is initialised at $z=40 d_{\mathrm{j}}$ on the jet axis where the mixture fraction is close to stoichiometry, the second stage noted above becomes different. Instead of the radial expansion, the flame expands rapidly in the vicinity of $\widetilde{Z}_{\text {st }}$ iso-line and then starts to propagate upstream immediately. As noted earlier in Fig. 12, this results in the larger slope for $z / d_{\mathrm{j}}$ vs $t^{*}$ curve compared to that for the flame ignited at $z=30 d_{\mathrm{j}}$. Similar behaviours are observed for other jet velocities. 


\section{Conclusion}

Steady and unsteady RANS simulation of turbulent lifted methane jet flames are conducted using a partially premixed combustion model involving unstrained premixed flamelets and presumed PDF method. The correlation between the mixture fraction and progress variable fluctuations is included in the analysis through a correlated joint PDF method. The contribution from non-premixed mode combustion is also included in the overall mean reaction rate modelling. These contributions appear in modular form in this approach allowing us to include and test one effect at a time. This modelling approach was developed in an earlier study and tested for undiluted hydrogen flames [46]. The agreement between the measured and computed flame brush structure and lift-off heights was shown to be very good for the hydrogen flame [46]. In this study, the abilities of this modelling approach to capture the undiluted and air-diluted methane jet lifted flames are tested as these flames involve relatively lower jet exit velocities compared to the earlier lifted hydrogen flames. The thermo-chemistry and its interaction with turbulence are well known to be different for methane and hydrogen mixtures. The ability of this combustion modelling approach, outlined in section 2 , is tested and validated without altering the combustion sub-modelling parameters used in [46]. The main findings of this study are summarised as follows.

- Various jet exit velocities and air-dilution levels are tested for model validation. The calculated lift-off heights, $L_{\mathrm{f}}$, agree very well with the measured values [48] for the range of conditions tested here. This agreement is found to range from excellent to very good when the effects of both Z-c correlation and non-premixed combustion are included while calculating the mean reaction rate using the model $\mathrm{D}$ in Table 1 . This clearly indicates that both 
of these two effects are important and required to capture the complex processes and their interactions involved at the stabilisation height of lifted flames.

- A systematic evaluation showed that the contributions from non-premixed combustion is predominantly negative to the mean reaction rate and thus shifts the flame brush leading edge downstream compared to the situation when these contributions are excluded. The effects of non-premixed combustion exists over flammable region as one would expect. This contributions is observed to be more significant for the undiluted flame, F0, because of relatively large $\widetilde{\chi}_{Z}$ values, resulting from mixing with entrained air, compared to that for the air-diluted flames.

- The Z-c correlation influences the flame stabilisation by redistributing the reaction rate inside the flame brush downstream of the leading edge. This results in changes in the flame-flow interaction causing two dominant effects on the flame brush. These effects are (1) the size of reaction zone with large reaction rate near $\widetilde{Z}_{\mathrm{st}}$ is reduced and (2) the two flame, lean and rich, branches downstream of the leading edge are squashed towards $\widetilde{Z}_{\text {st }}$ contour. The former effect increases the lift-off height whereas the latter effect decreases $L_{\mathrm{f}}$. The overall effect of the $Z-c$ correlation is a resultant of these two opposing effects and the later effect is observed to be dominant and its relative role increases with dilution level. The relatively increased influence of the latter effect in the air-diluted flames is because the flame brush is thicker allowing the correlation to affect a larger part of the flame.

- The air-dilution increases the lift-off height for a given jet velocity because 
of an increase in the stoichiometric mixture fraction value, which usually resides in regions with higher velocity. The offset between the flame leading edge and the main heat releasing zone is found to be larger in the highly diluted case as has been observed in experimental [11] and LES [18] studies.

- The unsteady RANS simulations show that the intense reaction zones of initial flame kernel moves towards its neighbouring (closest) stoichiometric position and then it propagates upstream along the stoichiometric isosurface until the final stabilisation height is reached for a given jet velocity.

Although the modelling frame work used here seems robust to calculate the flame lift-off heights over a wide range of flame and flow conditions for methane and hydrogen, the transient evolution of the flame leading edge does not agree with the measured evolution when the initial flame kernel is placed near stoichiometric iso-surface resulting from scalar mixing field solution. However, this agreement is found to be quite good when the initial kernel is placed in rich mixtures. The reason for this difference is unclear at this time, nevertheless one must recall the fact that the sample size for experimental analysis is only 10 movies. More experimental data would help to resolve this matter unambiguously. Also, this may indicate the limitation of the URANS methodology to capture the large scale dynamics and its interaction with evolving flame in a transient manner and advanced approaches such as LES would be helpful. Furthermore, the difference seen in the transient response in this study might be due to the simulations employing axisymmetric configuration which excludes a possible evolution of the flame leading in a third physical dimension. These points will be addressed in a future study. 


\section{Acknowledgement}

The supports of Cambridge Overseas Trust and China Scholarship Council are

acknowledged by ZC. SR and NS acknowledge the support of MHI, Takasago, Japan.

\section{References}

[1] N. Peters, F.A. Williams, AIAA J. 21 (1983) 423-429.

[2] J.E. Broadwell, W.J.A. Dahm, M.G. Mungal, Proc. Combust. Inst. 20 (1984) 303-310.

[3] K. Wohl, N.M. Kapp, C. Gazley, Symposium on Combustion and Flame, and Explosion Phenomena 3 (1949) 3-21.

[4] L. Vanquickenborne, A. van Tiggelen, Combust. Flame 10 (1966) 59-69.

[5] H. Eickhoff, B. Lenze, W. Leuckel, Proc. Combust. Inst. 20 (1984) 311-318.

[6] P.N. Kioni, B. Rogg, K.N.C. Bray, A. Liñán, Combust. Flame 95 (1993) 276-290.

[7] R.W. Schefer, P.J. Goix, Combust. Flame 112 (1998) 559-574.

[8] G.T. Kalghatgi, Combust. Sci. Technol. 41 (1984) 17-29.

[9] M.S. Mansour, Combust. Flame 133 (2003) 263-274.

[10] A. Cessou, C. Maurey, D. Stepowski, Combust. Flame 137 (2004) 458-477.

[11] L.K. Su, O.S. Sun, M.G. Mungal, Combust. Flame 144 (2006) 494-512. 
[12] D. Bradley, P.H. Gaskell, A.K.C. Lau, Proc. Combust. Inst. 23 (1990) 685692.

[13] C.M. Müller, H. Breitbach, N. Peters, Proc. Combust. Inst. 25 (1994) 1099 1106.

[14] V. Favier, L. Vervisch, Proc. Combust. Inst. 27 (1998) 1239-1245.

[15] Y. Mizobuchi, S. Tachibana, J. Shinio, S. Ogawa, T. Takeno, Proc. Combust. Inst. 29 (2002) 2009-2015.

[16] C.B. Devaud, K.N.C. Bray, Combust. Flame 132 (2003) 102-114.

[17] P. Domingo, L. Vervisch, K.N.C. Bray, Combust. Theory Model. 6 (2002) $529-551$.

[18] S.A. Ferraris, J.X. Wen, Combust. Flame 150 (2007) 320-339.

[19] S. Ruan, N. Swaminathan, Y. Mizobuchi, Combust. Sci. Technol. 186 (2014) 243-272.

[20] W.M. Pitts, Proc. Combust. Inst. 22 (1988) 809-816.

[21] N. Peters, Turbulent Combustion, Cambridge University Press, 2000.

[22] K.M. Lyons, Prog. Energy Combust. Sci. 33 (2007) 211-231.

[23] C.J. Lawn, Prog. Energy Combust. Sci. 35 (2009) 1-30.

[24] J. Buckmaster, Prog. Energy Combust. Sci. 28 (2002) 435-475.

[25] R.C. Miake-Lye, J.A. Hammer, Proc. Combust. Inst. 22 (1989) 817-824. 
[26] C.S. Yoo, R. Sankaran, J.H. Chen, J. Fluid Mech. 640 (2009) 453-481.

[27] K.A. Watson, K.M. Lyons, J.M. Donbar, C.D. Carter, Combust. Sci. Technol. 175 (2003) 649-664.

[28] A. Upatnieks, J.F. Driscoll, C.C. Rasmussen, S.L. Ceccio, Combust. Flame 138 (2004) 252-272.

[29] P. Domingo, L. Vervisch, J. Réveillon, Combust. Flame 140 (2005) 172-195.

[30] P. Domingo, L. Vervisch, D. Veynante, Combust. Flame 152 (2008) 415432.

[31] C.S. Yoo, E.S. Richardson, R. Sankaran, J.H. Chen, Proc. Combust. Inst. 33 (2011) 1619-1627.

[32] M. Chen, M. Herrmann, N. Peters, Proc. Combust. Inst. 28 (2000) 167-174.

[33] D. Bradley, P.H. Gaskell, X.J. Gu, Proc. Combust. Inst. 27 (1998) 11991206.

[34] C.Y. Ma, T. Mahmuda, M. Fairweather, E. Hampartsoumian, P.H. Gaskell, Combust. Flame 128 (2002) 60-73.

[35] L. Vervisch, R. Hauguel, P. Domingo, M. Rullaud, J. Turbulence 5 (2004) 004.

[36] A.W. Vreman, B.A. Albrecht, J.A. van Oijen, L.P.H. de Goey, R.J.M. Bastiaans, Combust. Flame 153 (2008) 394-416.

[37] J.-B. Michel, O. Colin, C. Angelberger, D. Veynante, Combust. Flame 156 (2009) 1318-1331. 
[38] I.S. Kim, E. Mastorakos, Proc. Combust. Inst. 30 (2005) 911-918.

[39] S. Navarro-Martinez, A. Kronenburg, Flow Turbulence Combust. 87 (2011) $377-406$.

[40] G. Lacaze, E. Richardson, T. Poinsot, Combust. Flame 156 (2009) 19932009.

[41] S. Donnerhack, N. Peters, Combust. Sci. Technol. 41 (1984) 101-108.

[42] N.A. Rokke, J.E. Hustad, O.K. Sonju, Combust. Flame 97 (1994) 88-106.

[43] W. J. S. Ramaekers, J. A. van Oijen, L. P. H. de Goey, Flow Turbulence Combust. 84 (2010) 439-458.

[44] L. M. Verhoeven, W. J. S. Ramaekers, J. A. van Oijen, L. P. H. de Goey, Combust. Flame 159 (2012) 230-241.

[45] Y. Mizobuchi, J. Shinjo, S. Ogawa, T. Takeno, Proc. Combust. Inst. 30 (2005) 611-619.

[46] S. Ruan, N. Swaminathan, O.R. Darbyshire, Combust. Theory Model. 18 (2014) 295-329.

[47] O.R. Darbyshire, N. Swaminathan, Combust. Sci. Technol. 184 (2012) 2036-2067.

[48] S.F. Ahmed, E. Mastorakos, Combust. Flame 146 (2006) 215-231.

[49] S. Ruan, N. Swaminathan, K.N.C. Bray, Y. Mizobuchi, T. Takeno, Combust. Flame 159 (2012) 591-608. 
[50] T. Mantel, R.W. Bilger, Combust. Sci. Technol. 110-111 (1995) 393-417.

[51] N. Swaminathan, K.N.C. Bray, Combust. Flame 143 (2005) 549-565.

[52] N. Chakraborty, M. Champion, A. Mura, N. Swaminathan, in: N. Swaminathan, K.N.C. Bray (Eds.), Turbulent Premixed Flames, Cambridge University Press, New York, 2011, pp. 74-102.

[53] H. Kolla, J.W. Rogerson, N. Chakraborty, N. Swaminathan, Combust. Sci. Technol. 181 (2009) 518-535.

[54] O.R. Darbyshire, N. Swaminathan, S. Hochgreb, Combust. Sci. Technol. 182 (2010) 1141-1170.

[55] R.W. Bilger, S.H. Stårner, R.J. Kee, Combust. Flame 80 (1990) 135-149.

[56] B. Fiorina, R. Baron, O. Gicquel, D. Thevenin, S. Carpentier, N. Darabiha, Combust. Theory Model. 7 (2003) 449-470.

[57] K.N.C. Bray, P. Domingo, L. Vervisch, Combust. Flame 141 (2005) 431437.

[58] M. D. Smooke, V. Giovangigli, in: M. D. Smooke (Ed.), Reduced Kinetic Mechanisms and Asymptotic Approximations for Methane-air Flames, volume 384 of Lecture Notes in Physics, Springer-Verlag, Berlin, 1991, pp. $1-28$.

[59] P.-D. Nguyen, L. Vervisch, V. Subramanian, P. Domingo, Combust. Flame 157 (2010) 43-61. 
[60] L. Vervisch, V. Moureau, P. Domingo, D. Veynante, in: N. Swaminathan, K.N.C. Bray (Eds.), Turbulent premixed flames, Cambridge University Press, New York, 2011, pp. 60-74.

[61] D.C. Wilcox, Turbulence Modeling for CFD, DCW Industries, Inc., 2006.

[62] W.P. Jones, M. Kakhi, Combust. Flame 115 (1998) 210-229.

[63] S.R. Tieszen, D.W. Stamps, T.J. O’Hern, Combust. Flame 106 (1996) 442466.

[64] C.D. Richards, W.M. Pitts, J. Fluid Mech. 254 (1993) 417-435.

[65] H. Kolla, N. Swaminathan, J. Combust. 2011 (2011).

[66] H. Kolla, N. Swaminathan, Combust. Flame 157 (2010) 943-954.

[67] H. Kolla, N. Swaminathan, Combust. Flame 157 (2010) 1274-1289.

[68] H. Kolla, J.W. Rogerson, N. Swaminathan, Combust. Sci. Technol. 182 (2010) 284-308.

[69] A. Joedicke, N. Peters, M. Mansour, Proc. Combust. Inst. 30 (2005) 901909.

[70] S.H. Stårner, R.W. Bilger, J.H. Frank, D.F. Marran, M.B. Long, Combust. Flame 107 (1996) 307-313.

[71] R.W. Schefer, M. Namazian, J. Kelly, Combust. Flame 99 (1994) 75-86. 


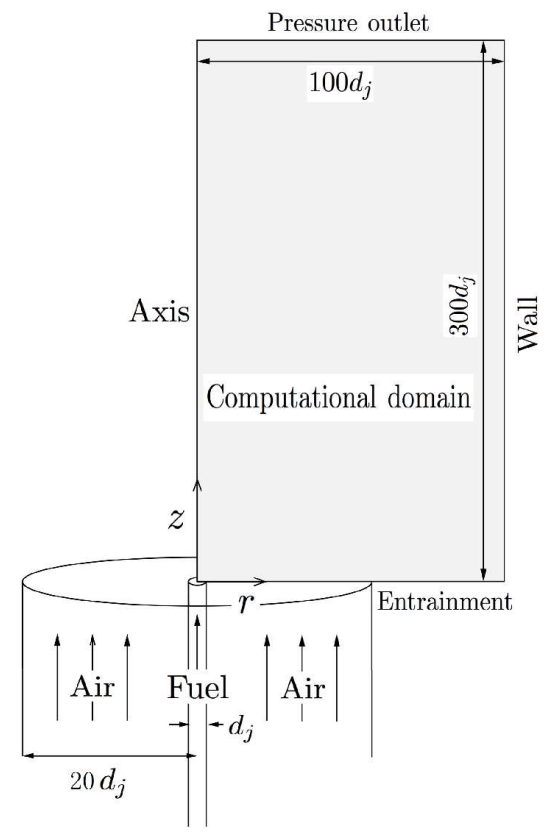

(a)
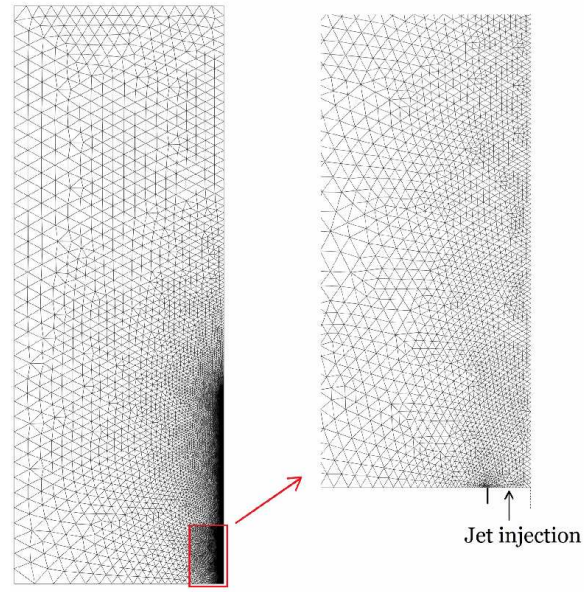

(b)

Figure 1: A schematic of the burner setup for experiments in [48] and computations. Computational domain is shown in (a) and a typical numerical grid is shown in (b). 

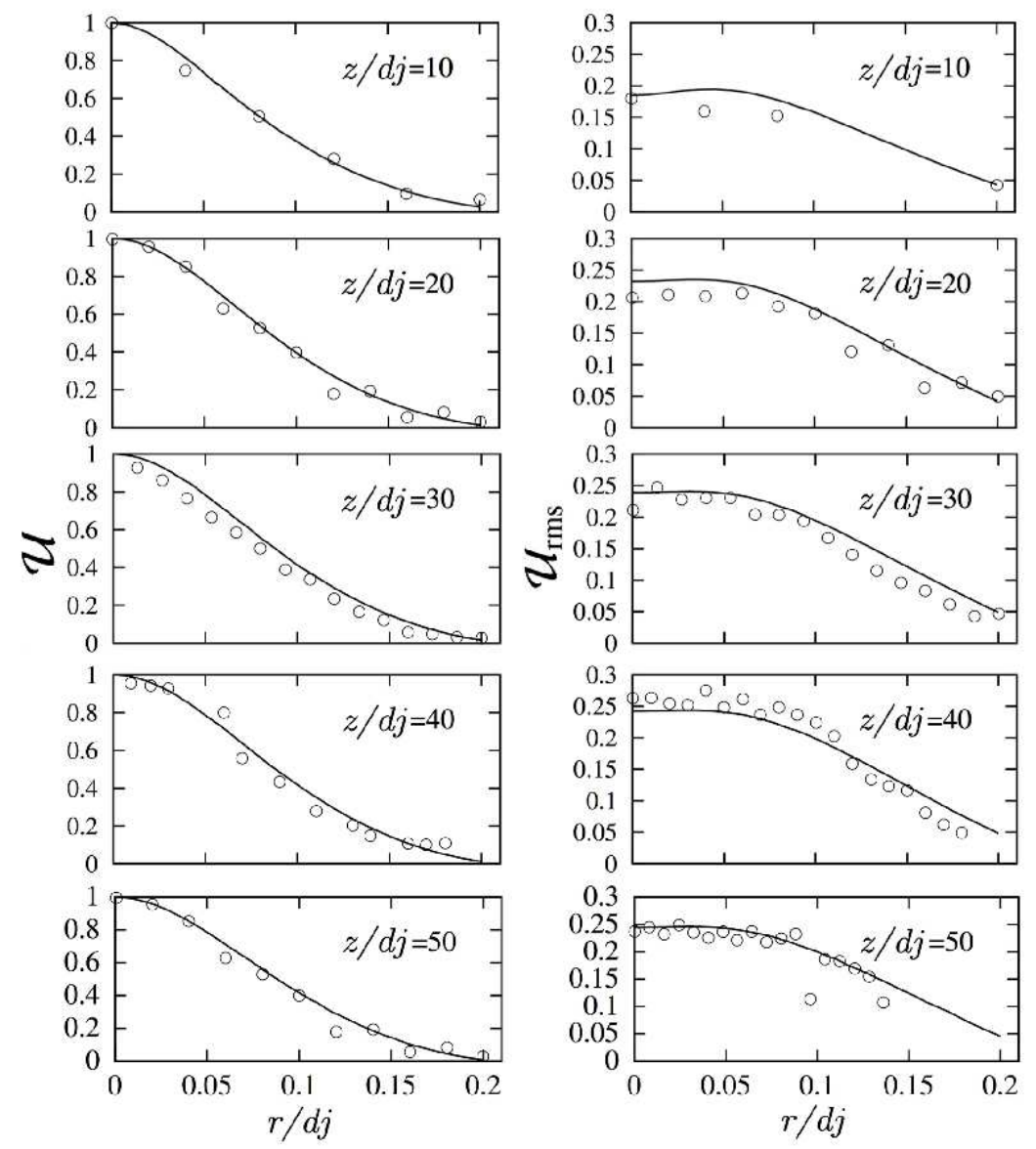

Figure 2: Comparison of computed (-) and measured [48] (०००) radial variation of mean axial velocity and turbulence r.m.s. values. The values are normalised as noted in the text. Air injection velocity is $U_{\mathrm{j}}=21 \mathrm{~m} / \mathrm{s}$ and air co-flow velocity is $U_{\mathrm{c}}=0.1 \mathrm{~m} / \mathrm{s}$. 


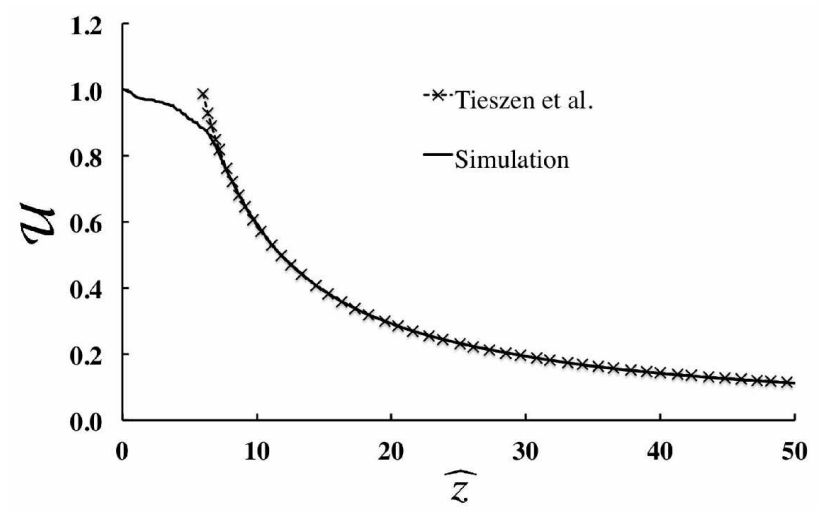

Figure 3: The axial variation of scaled centreline velocity, $\mathcal{U}_{c l}$ computed in this study is compared to the empirical relationship established in [63].

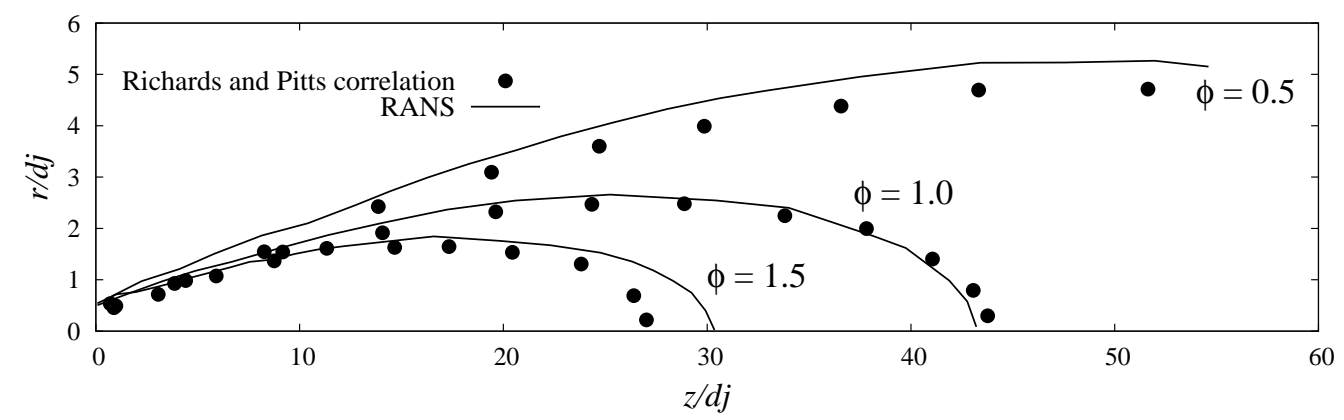

Figure 4: Comparison equivalence ratio contours obtained using empirical relation [64] in Eq. (24) and from RANS simulation for the case F3, 30\% air-diluted $\mathrm{CH}_{4}$ jet, with $U_{\mathrm{j}}=25.5 \mathrm{~m} / \mathrm{s}$ and $U_{\mathrm{c}}=0.1 \mathrm{~m} / \mathrm{s}$. 


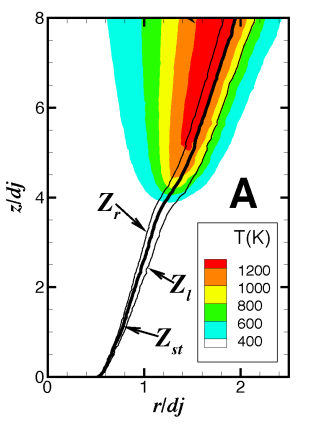

(a)

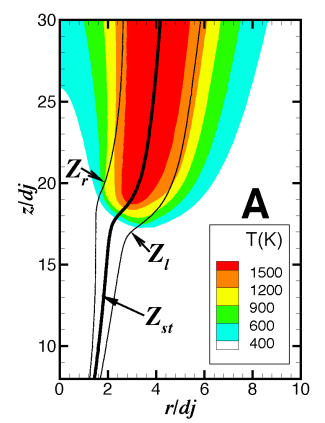

(e)

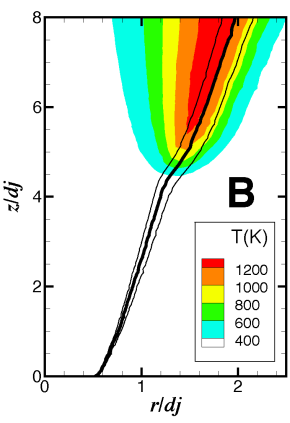

(b)

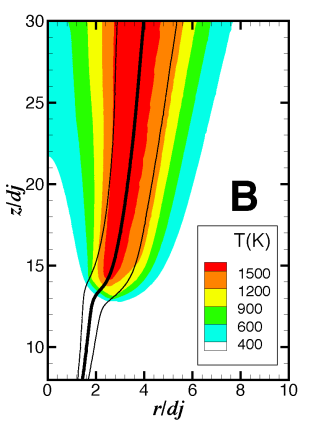

(f)

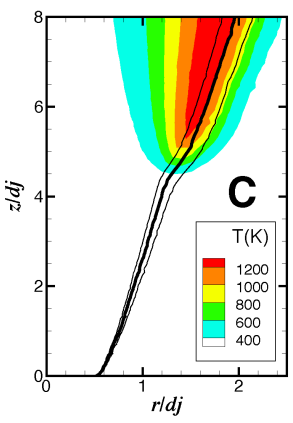

(c)

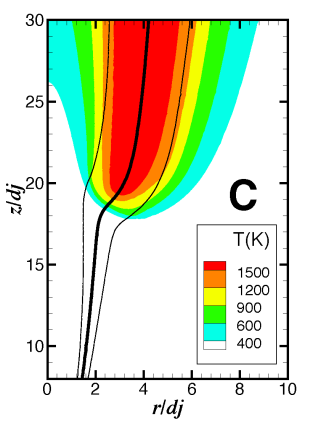

(g)

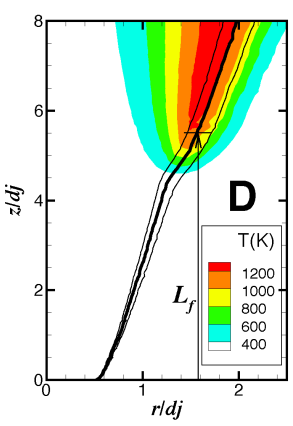

(d)

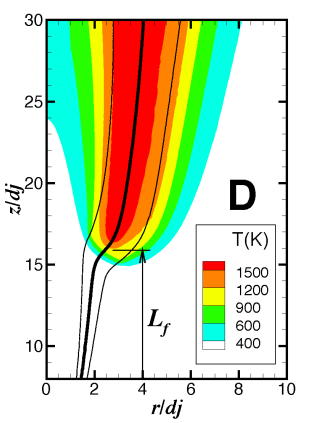

(h)

Figure 5: Temperature (dashed line) and mixture fraction (line) contours for flame F0 (top row) and F4 (bottom row). The jet exit velocity for these two cases is $U_{\mathrm{j}}=16 \mathrm{~m} / \mathrm{s}$. 


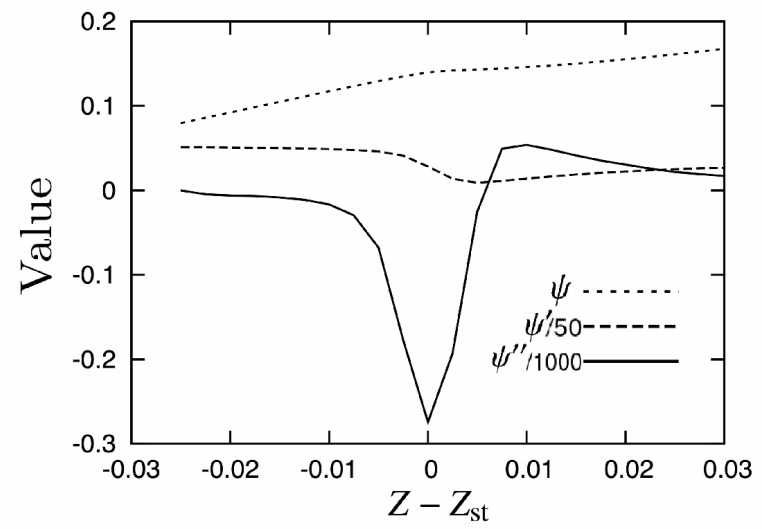

Figure 6: Variations of $\psi^{\mathrm{Eq}}, \psi^{\prime}=d \psi^{\mathrm{Eq}} / d Z$ and $\psi^{\prime \prime}=d^{2} \psi^{\mathrm{Eq}} / d Z^{2}$ with $Z$ in the vicinity of $Z_{\mathrm{st}}$. 

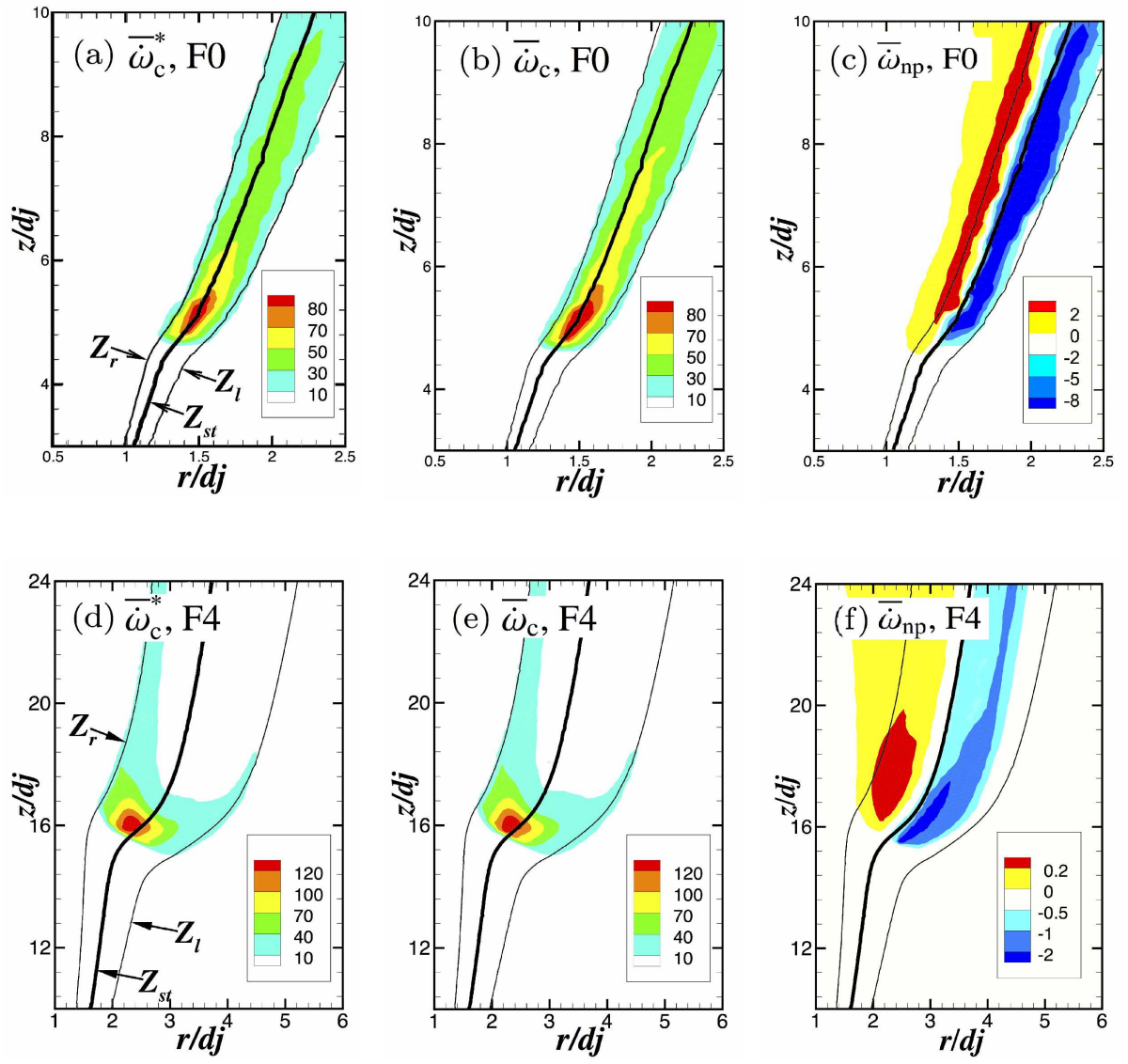

Figure 7: Spatial variation of mean reaction rate, $\overline{\dot{\omega}}_{c}^{*}$, and its components $\overline{\dot{\omega}}_{c}$ and $\overline{\dot{\omega}}_{\text {np }}\left(\mathrm{kg} / \mathrm{m}^{3} / \mathrm{s}\right)$ in flames F0 and F4. The jet exit velocity for these two flames is $U_{\mathrm{j}}=16 \mathrm{~m} / \mathrm{s}$. 

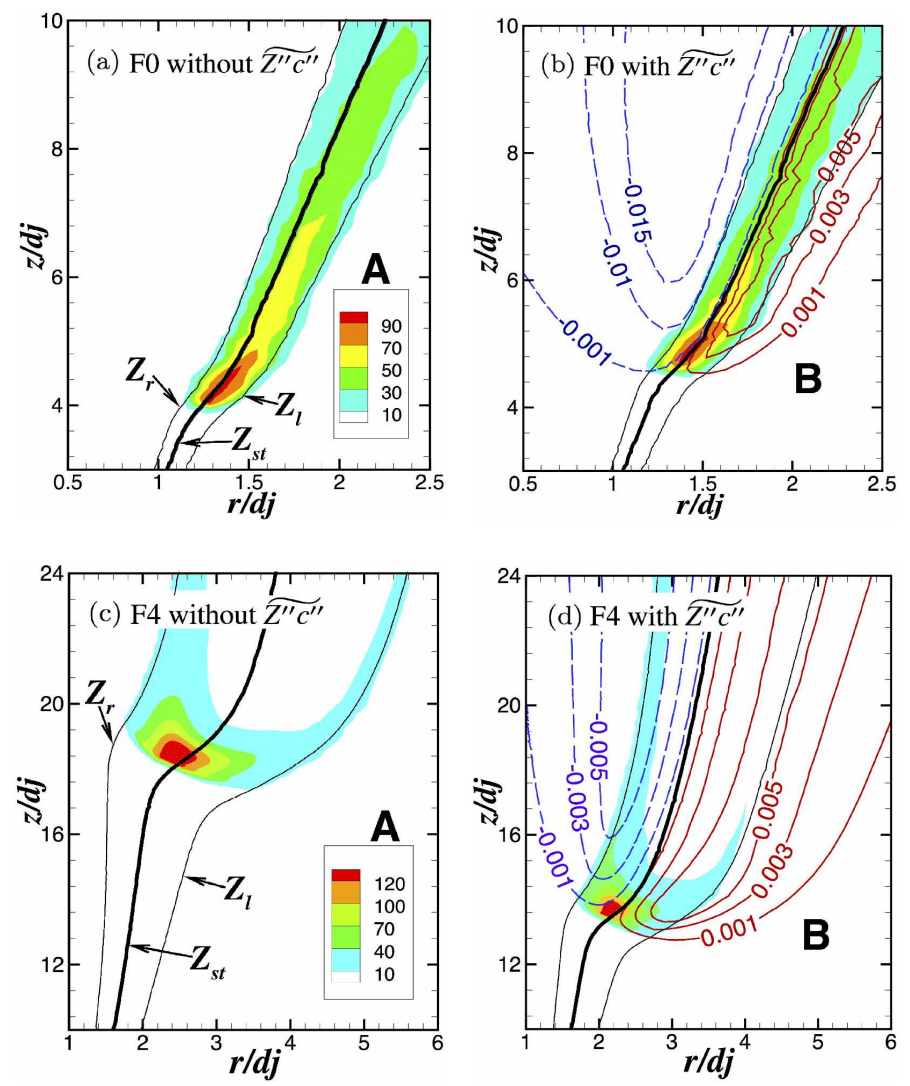

Figure 8: Effects of $Z-c$ correlation on the premixed mean reaction rate, $\overline{\dot{\omega}}_{c}$. The mean reaction rate in $\mathrm{kg} / \mathrm{m}^{3} / \mathrm{s}$ is shown using pseudo colours and the iso-contours of $\widetilde{Z^{\prime \prime} c^{\prime \prime}}$ are shown as lines, solid line is for $\widetilde{Z^{\prime \prime} c^{\prime \prime}}>0$ and dashed line is for $\widetilde{Z^{\prime \prime} c^{\prime \prime}}<0$. 

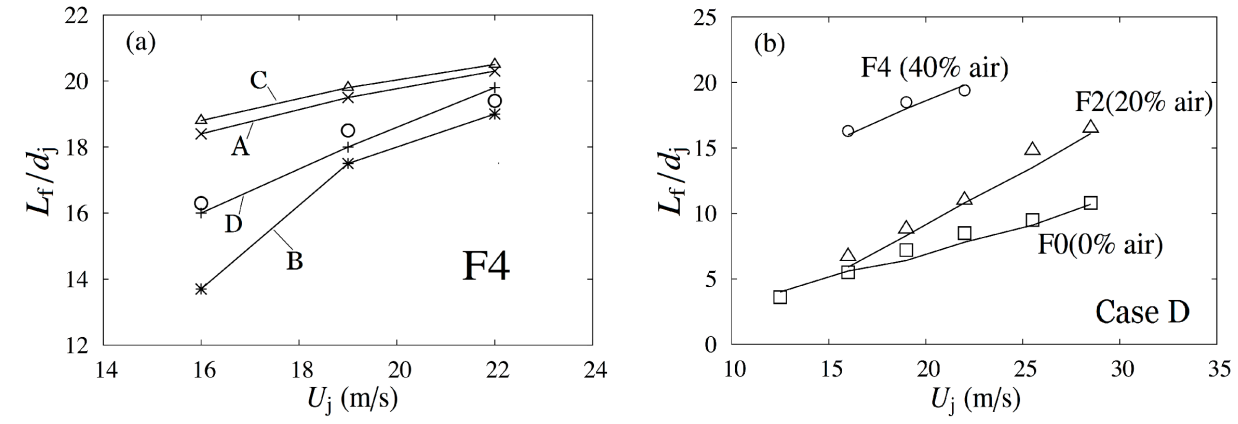

Figure 9: Computed flame lift-off height is compared to the measured [48] values for various jet velocities.
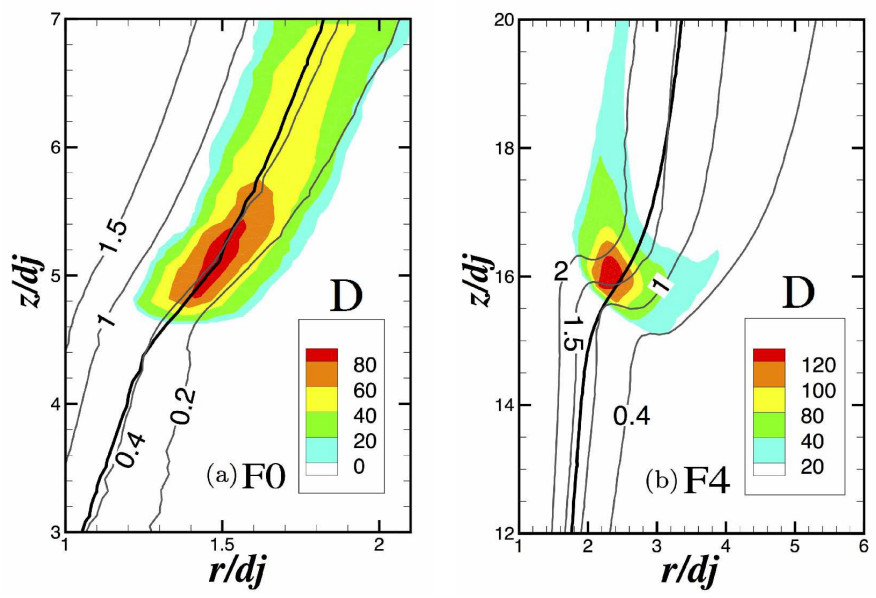

Figure 10: Contours of $\overline{\dot{\omega}_{c}^{*}}$ in $\mathrm{kg} / \mathrm{m}^{3} / \mathrm{s}$ (colour map) and $\widetilde{U}(\mathrm{~m} / \mathrm{s})$ (thin lines). The black bold line is for $\widetilde{Z}_{\mathrm{st}}$. The jet exit velocity for these two flames is $U_{\mathrm{j}}=16 \mathrm{~m} / \mathrm{s}$. 

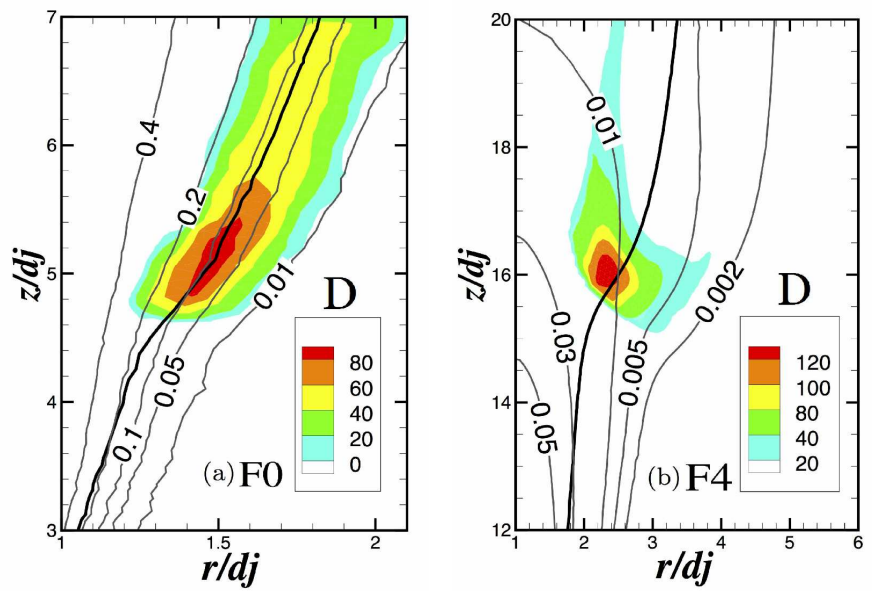

Figure 11: Contours of $\overline{\dot{\omega}_{c}^{*}}$ in $\mathrm{kg} / \mathrm{m}^{3} / \mathrm{s}$ (colour map) and $\widetilde{\chi}_{z} / \widetilde{\chi}_{z, q}$ (thin line). The black bold line is for $\widetilde{Z}_{s t}$. The jet exit velocity for these two flames is $U_{\mathrm{j}}=16 \mathrm{~m} / \mathrm{s}$. 

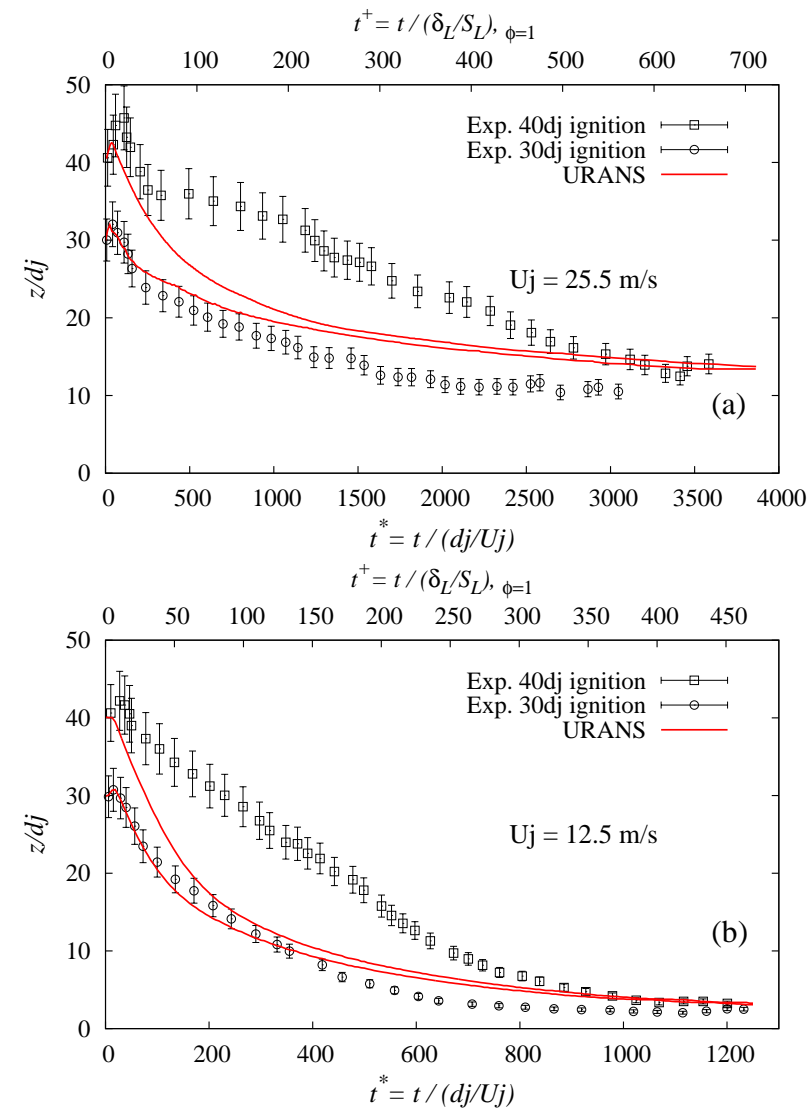

Figure 12: Temporal variation of axial location of the flame edge, marked using the leading edge of $\widetilde{T}=1200 \mathrm{~K}$ contour. The instant $t=0$ corresponds to ignition. The error bar corresponds to $9 \%$ maximum error reported in [48]. 


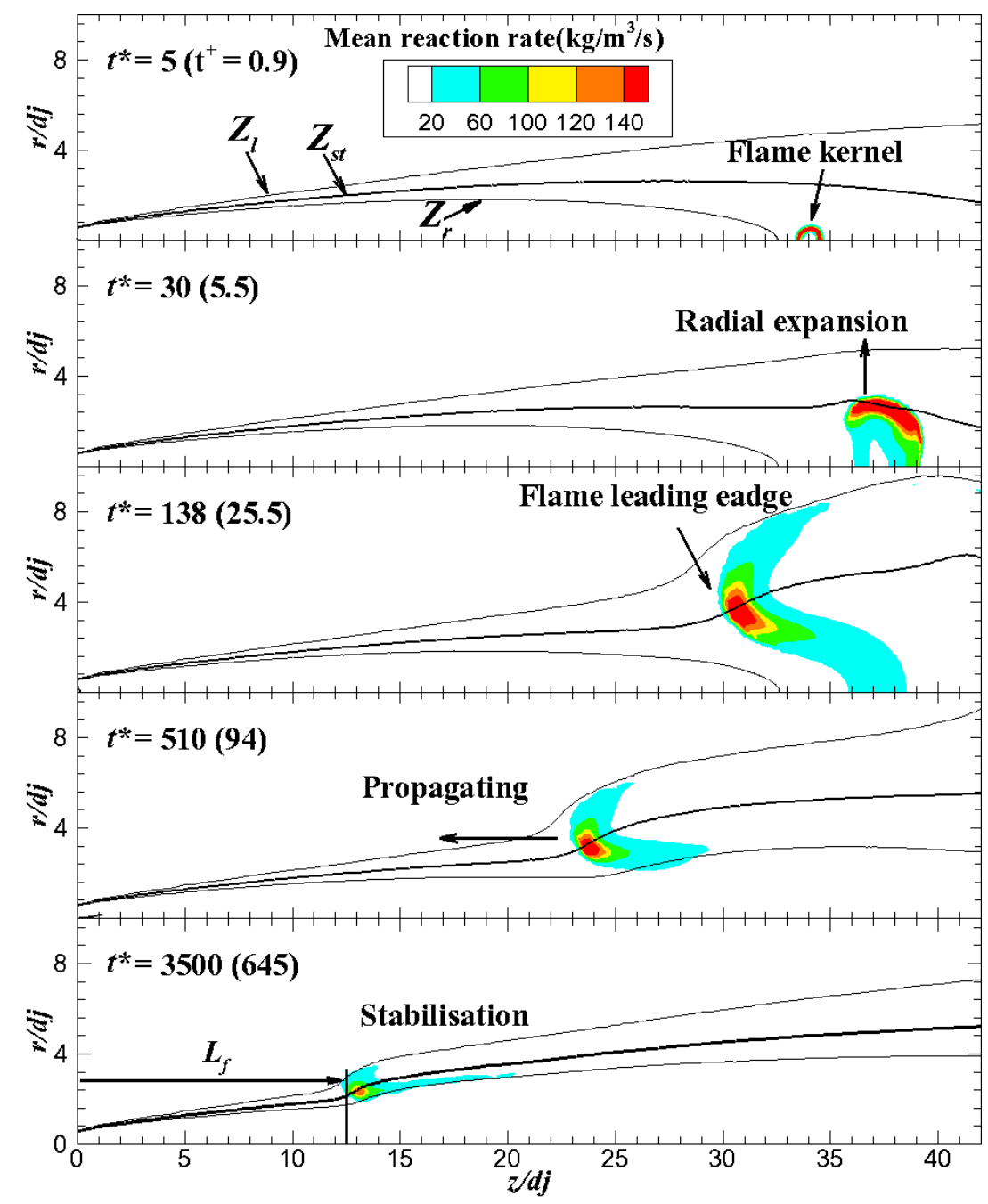

Figure 13: The flame brush evolution computed using model D for flame F3 (30\% air-dilution) towards its stabilisation height from its initial kernel location. 